\title{
Combination preconditioning of saddle point systems for positive definiteness*
}

\author{
J. Pestana*and A. J. Wathen \\ Mathematical Institute, University of Oxford, 24-29 St Giles', Oxford, OX1 3LB, UK
}

\begin{abstract}
SUMMARY
Amongst recent contributions to preconditioning methods for saddle point systems, standard iterative methods in nonstandard inner products have been usefully employed. Krzyżanowski (Numer. Linear Algebra Appl. 2011; 18:123-140) identified a two-parameter family of preconditioners in this context and Stoll and Wathen (SIAM J. Matrix Anal. Appl. 2008; 30:582-608) introduced combination preconditioning, where two preconditioners, self-adjoint with respect to different inner products, can lead to further preconditioners and associated bilinear forms or inner products. Preconditioners that render the preconditioned saddle point matrix nonsymmetric but self-adjoint with respect to a nonstandard inner product always allow a MINREStype method ( $\mathcal{W}$-PMINRES) to be applied in the relevant inner product. If the preconditioned matrix is also positive definite with respect to the inner product a more efficient CG-like method ( $\mathcal{W}$-PCG) can be reliably used. We establish eigenvalue expressions for Krzyżanowski preconditioners and show that for a specific choice of parameters, although the Krzyżanowski preconditioned saddle point matrix is self-adjoint with respect to an inner product, it is never positive definite. We provide explicit expressions for the combination of certain preconditioners and prove the rather counterintuitive result that the combination of two specific preconditioners for which only $\mathcal{W}$-PMINRES can be reliably used leads to a preconditioner for which, for certain parameter choices, $\mathcal{W}$-PCG is reliably applicable. That is, combining two indefinite preconditioners can lead to a positive definite preconditioner. This combination preconditioner outperforms either of the two preconditioners from which it is formed for a number of test problems. Copyright (c) 0000 John Wiley \& Sons, Ltd.
\end{abstract}

Received ...

KEY WORDS: iterative solvers, nonstandard inner products, preconditioning, saddle point problems

\section{INTRODUCTION}

Consider the real symmetric saddle point system

$$
\mathcal{A} x=\left[\begin{array}{cc}
A & B^{T} \\
B & -C
\end{array}\right] x=b,
$$

where $A \in \mathbb{R}^{n \times n}$ is symmetric positive definite, $C \in \mathbb{R}^{m \times m}$ is symmetric positive semidefinite, $B$ has full rank and $m \leq n$. Under these assumptions $\mathcal{A}$ is invertible [?, Theorem 3.1]. Such systems arise in a vast number of applications including constrained optimization, computational

\footnotetext{
*This is the accepted version of the following article: J. Pestana and A. J. Wathen. Combination preconditioning of saddle point systems for positive definiteness. Numerical Linear Algebra with Applications, 20:785-808. 2015., which has been published in final form at http://onlinelibrary.wiley.com/doi/10.1002/nla.1843/full.

${ }^{*}$ Correspondence to: J. Pestana, Mathematical Institute, University of Oxford, 2429 St Giles', Oxford, OX1 3LB, UK.

E-mail: pestana@maths.ox.ac.uk 
fluid dynamics and mixed finite element discretizations of elliptic PDEs [?, Section 2]. Often $\mathcal{A}$ is large and sparse and it is natural in these instances to solve the saddle point system by preconditioned Krylov subspace methods.

Many preconditioners $\mathcal{P} \in \mathbb{R}^{(n+m) \times(n+m)}$ for saddle point problems have been proposed, surveys of which can be found in, for example, Benzi, Golub and Liesen [?] and Benzi and Wathen [?]. If $\mathcal{P}$ is symmetric positive definite the system can be solved by preconditioned MINRES. However, many effective preconditioners are nonsymmetric or symmetric indefinite. Although it is possible to apply a nonsymmetric Krylov method to the preconditioned system in this situation, it may be appealing to turn instead to a Krylov method in a nonstandard inner product $\langle x, y\rangle_{\mathcal{W}}=x^{T} \mathcal{W} y$, where $\mathcal{W} \in \mathbb{R}^{(n+m) \times(n+m)}$ is symmetric positive definite. For certain preconditioners, $\mathcal{P}^{-1} \mathcal{A}$ is self-adjoint with respect to a known inner product $\langle\cdot, \cdot\rangle_{\mathcal{W}}$, i.e., $\left\langle\mathcal{P}^{-1} \mathcal{A} x, y\right\rangle_{\mathcal{W}}=\left\langle x, \mathcal{P}^{-1} \mathcal{A} y\right\rangle_{\mathcal{W}}$ for all $x, y \in \mathbb{R}^{n+m}$ in which case MINRES in $\langle\cdot, \cdot\rangle_{\mathcal{W}}$ can be used. If, in addition, $\left\langle\mathcal{P}^{-1} \mathcal{A} x, x\right\rangle_{\mathcal{W}}>0$ for all nonzero $x \in \mathbb{R}^{n+m}$, so that $\mathcal{P}^{-1} \mathcal{A}$ is $\mathcal{W}$-positive definite, a conjugate gradient method in $\langle\cdot, \cdot\rangle_{\mathcal{W}}$ can be applied instead. The attraction of these nonstandard methods is that they minimize the residual or error with respect to a norm and require only three-term recurrences. One potential disadvantage is that computations with $\langle\cdot, \cdot\rangle_{\mathcal{W}}$ must be performed at each iteration. Often, however, the inner product depends on the same blocks as the preconditioner, that are generally sparse, and computational savings can be made by careful consideration of the operations involved [?]. We note that an alternative Krylov subspace method when the preconditioned saddle point matrix is self-adjoint with respect to a symmetric bilinear form (that is not positive definite) is SQMR [?].

There exist several preconditioners for which the preconditioned linear system can be made selfadjoint with respect to an inner product (see, for example, [?, ?, ?, ?, ?, ?, ?, ?]). The BramblePasciak (BP) [?] construction is a particularly well used example. Recently, Stoll and Wathen [?] showed that two such preconditioners could be combined to give a new preconditioner such that the preconditioned saddle point matrix is self-adjoint with respect to a symmetric bilinear form that can, in many cases, be made an inner product. They also introduced the Bramble-Pasciak ${ }^{+}$ $\left(\mathrm{BP}^{+}\right)$preconditioner. One might expect that if one or both of the preconditioned linear systems $\mathcal{P}^{-1} \mathcal{A}$ is positive definite with respect to an inner product then the combination preconditioner could either inherit the positive definiteness property or lose it. What is perhaps more surprising is that a combination preconditioner can be constructed for which the combination preconditioned saddle point matrix is positive definite with respect to an inner product from two preconditioners for each of which $\mathcal{P}^{-1} \mathcal{A}$ is indefinite with respect to an inner product. This combination preconditioner is described and investigated here.

The remainder of the paper is structured as follows. We detail pertinent concepts related to symmetric bilinear forms and inner products in Section 2 and describe in Section 3 the nonstandard methods we will use, $\mathcal{W}$-PCG and $\mathcal{W}$-PMINRES. An overview of some existing preconditioners for saddle point matrices is provided in Section 4, where we also show that a preconditioner suggested by Krzyżanowski [?], that we call the "Schöberl-Zulehner" " $\left(\mathrm{SZ}^{+}\right)$preconditioner, is such that the preconditioned coefficient matrix is indefinite with respect to an inner product. Section 5 describes combination preconditioning and proposes two combination preconditioners, each of which is built from preconditioners for which $\mathcal{P}^{-1} \mathcal{A}$ is indefinite. The $\mathrm{BP}^{+}-\mathrm{SZ}^{+}$combination preconditioner is also indefinite with respect to an inner product but for the $\mathrm{BP}^{+}-\mathrm{BD}$ combination preconditioner definiteness can be achieved, where BD denotes a block diagonal preconditioner that is described below. Numerical results for the latter combination preconditioner are given in Section 6 with conclusions made in Section 7.

Throughout, we denote by $S=B A^{-1} B^{T}+C$ the (negative) Schur complement. We assume that approximations $A_{0}$ of $A$, and $S_{0}$ of $S$, are symmetric positive definite. Throughout, we use $I$-CG and $I$-MINRES for the standard conjugate gradient and MINRES methods and denote the Euclidean norm by the $I$-norm for consistency. When applied to matrices, $>$ represents the positive semi-definite ordering, so that $A>A_{0}$ means that $A-A_{0}$ is positive definite. 


\section{SELF-ADJOINTNESS}

In this section we define symmetric bilinear forms and inner products and present background material necessary to understand Krylov methods in nonstandard inner products. We consider only symmetric bilinear forms on $\mathbb{R}^{n}$ but the extension to symmetric bilinear, or Hermitian sesquilinear, forms on $\mathbb{C}^{n}$ is straightforward.

A nondegenerate symmetric bilinear form [?, Section 2.1] on $\mathbb{R}^{n}$ satisfies

$$
\langle x, y\rangle_{\mathcal{W}}=y^{T} \mathcal{W} x
$$

for all $x, y \in \mathbb{R}^{n}$ and an invertible symmetric matrix $\mathcal{W} \in \mathbb{R}^{n \times n}$. An inner product on $\mathbb{R}^{n}$ is a symmetric bilinear form for which $\mathcal{W} \in \mathbb{R}^{n \times n}$ is symmetric positive definite.

In the case that (2) is an inner product we can associate with it a $\mathcal{W}$-(vector) norm for $x \in \mathbb{R}^{n}$,

$$
\|x\|_{\mathcal{W}}=\sqrt{\langle x, x\rangle_{\mathcal{W}}}
$$

and a $\mathcal{W}$-matrix norm for $B \in \mathbb{R}^{n \times n}$,

$$
\|B\|_{\mathcal{W}}=\max _{x \neq 0} \frac{\|B x\|_{\mathcal{W}}}{\|x\|_{\mathcal{W}}} .
$$

A matrix $B \in \mathbb{R}^{n \times n}$ is $\mathcal{W}$-self-adjoint, i.e., self-adjoint with respect to $\langle\cdot, \cdot\rangle_{\mathcal{W}}$, if and only if $\langle B x, y\rangle_{\mathcal{W}}=\langle x, B y\rangle_{\mathcal{W}}$, or $y^{T} B^{T} \mathcal{W} x=y^{T} \mathcal{W} B x$, for all $x, y \in \mathbb{R}^{n}$. Thus, an equivalent condition for self-adjointness is that

$$
\mathcal{W} B=B^{T} \mathcal{W},
$$

which shows that we require that $\mathcal{W} B$ is symmetric. A matrix $B$ is self-adjoint with respect to some inner product if and only if it is diagonalizable with real eigenvalues (see, for example, [?]).

A $\mathcal{W}$-self-adjoint matrix $B$ is $\mathcal{W}$-positive definite if and only if $\langle B x, x\rangle_{\mathcal{W}}>0 \quad \forall x \in \mathbb{R}^{n}, x \neq 0$, which is equivalent to requiring that

$$
x^{T} \mathcal{W} B x>0
$$

for all nonzero $x$. Since $B$ is self-adjoint with respect to $\langle\cdot, \cdot\rangle_{\mathcal{W}}, \mathcal{W} B$ is symmetric and it follows that the eigenvalues of a $\mathcal{W}$-positive definite, $\mathcal{W}$-self-adjoint matrix are not only real but positive.

\section{KRYLOV SUBSPACE METHODS IN NONSTANDARD INNER PRODUCTS}

We now outline two short-term recurrence methods in nonstandard inner products for the preconditioned linear system

$$
\mathcal{P}^{-1} \mathcal{A} x=\mathcal{P}^{-1} b,
$$

where $\mathcal{P}^{-1} \mathcal{A}$ is self-adjoint with respect to an inner product $\langle\cdot, \cdot\rangle_{\mathcal{W}}$. Although we focus on left preconditioning here, methods for $\mathcal{W}$-self-adjoint right preconditioned systems can be similarly derived.

\section{1. $\mathcal{W}-P C G$}

Although the conjugate gradient method is typically presented with the Euclidean inner product the original method of Hestenes and Stiefel [?] is derived in a general inner product and numerous examples of conjugate gradient methods in nonstandard inner products can be found in the literature $[?, ?, ?, ?, ?, ?, ?]$. The reliable application of the $\mathcal{W}$-PCG method in Algorithm 1 requires that $\mathcal{P}^{-1} \mathcal{A}$ be self-adjoint and positive definite with respect to an inner product $\langle\cdot, \cdot\rangle_{\mathcal{W}}$.

The $\mathcal{W}$-PCG method minimizes the error $e_{k}=x-x_{k}$ over $x_{0}+\mathcal{K}_{k}\left(\mathcal{P}^{-1} \mathcal{A}, \mathcal{P}^{-1} r_{0}\right)$, i.e.,

$$
x_{k}=\arg \min _{y \in x_{0}+\mathcal{K}_{k}\left(\mathcal{P}^{-1} \mathcal{A}, \mathcal{P}^{-1} r_{0}\right)}\|x-y\|_{\mathcal{W P}^{-1} \mathcal{A}},
$$

which can be bounded similarly to the error for $I$-CG. 


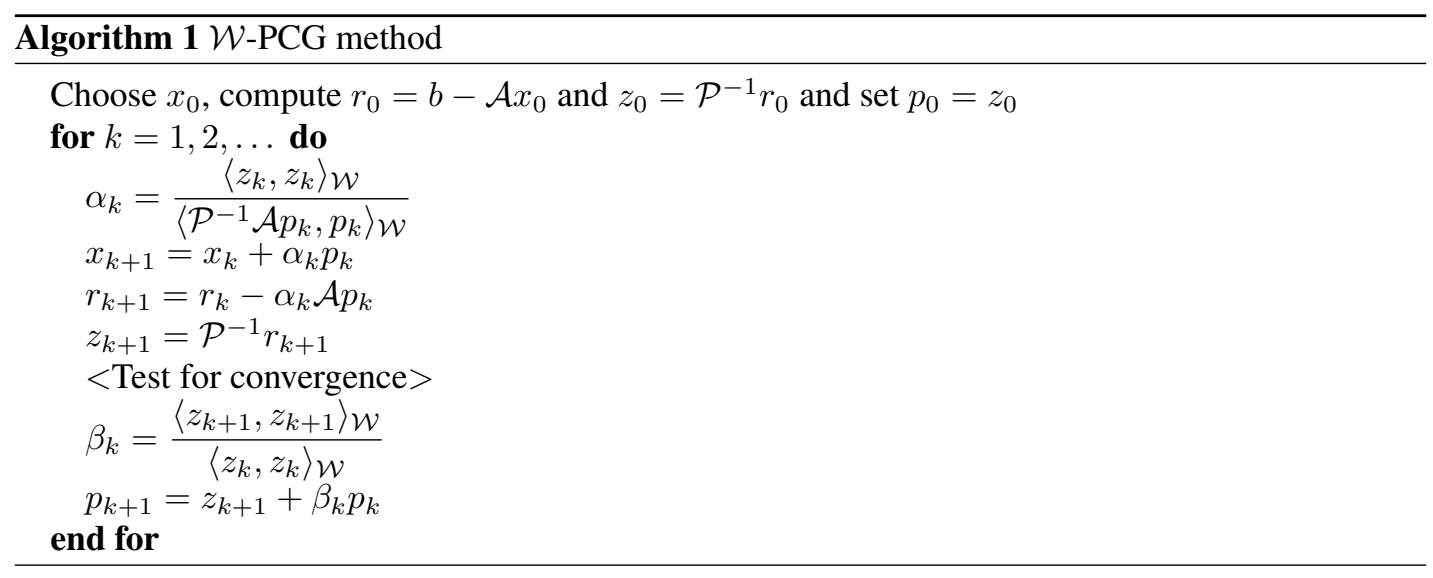

\section{Lemma 1}

The error, $e_{k}$, of the $k$ th $\mathcal{W}$-PCG iterate is given by

$$
\frac{\left\|e_{k}\right\|_{\mathcal{W P}^{-1} \mathcal{A}}}{\left\|e_{0}\right\|_{\mathcal{W} \mathcal{P}^{-1} \mathcal{A}}} \leq \min _{p \in \Pi_{k}, p(0)=1} \max _{\lambda \in \sigma\left(\mathcal{P}^{-1} \mathcal{A}\right)}|p(\lambda)|
$$

Proof

Since $\mathcal{P}^{-1} A$ is self-adjoint with respect to an inner product it is diagonalizable. As a consequence, the proof is almost identical to that for the conjugate gradient method in the Euclidean inner product in, for example, [?, Section 2.1.1].

From Lemma 1 we discern that when a preconditioned coefficient matrix has eigenvalues for which polynomials, satisfying $p(0)=1$, can be found that are small at each eigenvalue, convergence will be rapid. This is the motivation behind choosing preconditioners for which $\mathcal{P}^{-1} \mathcal{A}$ has nicely distributed eigenvalues, similarly to the standard preconditioned conjugate gradient method.

\section{2. $\mathcal{W}$-PMINRES}

The original $I$-MINRES algorithm of Paige and Saunders [?] minimizes the $I$-norm of the residual. Analogously, the $\mathcal{W}$-PMINRES method in Algorithm (2) minimizes

$$
\left\|\mathcal{P}^{-1} r_{k}\right\|_{\mathcal{W}}=\min _{y \in x_{0}+\mathcal{K}_{k}\left(\mathcal{P}^{-1} \mathcal{A}, \mathcal{P}^{-1} r_{0}\right)}\left\|\mathcal{P}^{-1}(b-\mathcal{A} y)\right\|_{\mathcal{W}}
$$

Each iteration of the $\mathcal{W}$-PMINRES method is more expensive than that of the $\mathcal{W}$-PCG method but its reliable application requires only that $\mathcal{P}^{-1} \mathcal{A}$ is self-adjoint with respect to the inner product $\langle\cdot, \cdot\rangle_{\mathcal{W}}$.

The minimization property again allows us to bound the preconditioned residuals of $\mathcal{W}$ PMINRES [?, ?].

Lemma 2

Let $\mathcal{P}^{-1} \mathcal{A}$ be self-adjoint with respect to $\langle\cdot, \cdot\rangle_{\mathcal{W}}$. Then, $\mathcal{W}$-preconditioned MR residuals are bounded by

$$
\frac{\left\|\mathcal{P}^{-1} r_{k}\right\|_{\mathcal{W}}}{\left\|\mathcal{P}^{-1} r_{0}\right\|_{\mathcal{W}}} \min _{p \in \Pi_{k}, p(0)=1} \max _{\lambda \in \sigma\left(\mathcal{P}^{-1} \mathcal{A}\right)}|p(\lambda)|
$$

Proof

See [?, Section 3] or [?, Lemma 1].

Lemma 2 shows that preconditioners that make $\mathcal{P}^{-1} \mathcal{A}$ self-adjoint with respect to an inner product with which it is practical to work and achieve a nice eigenvalue distribution will define an effective $\mathcal{W}$-PMINRES method for (1). 


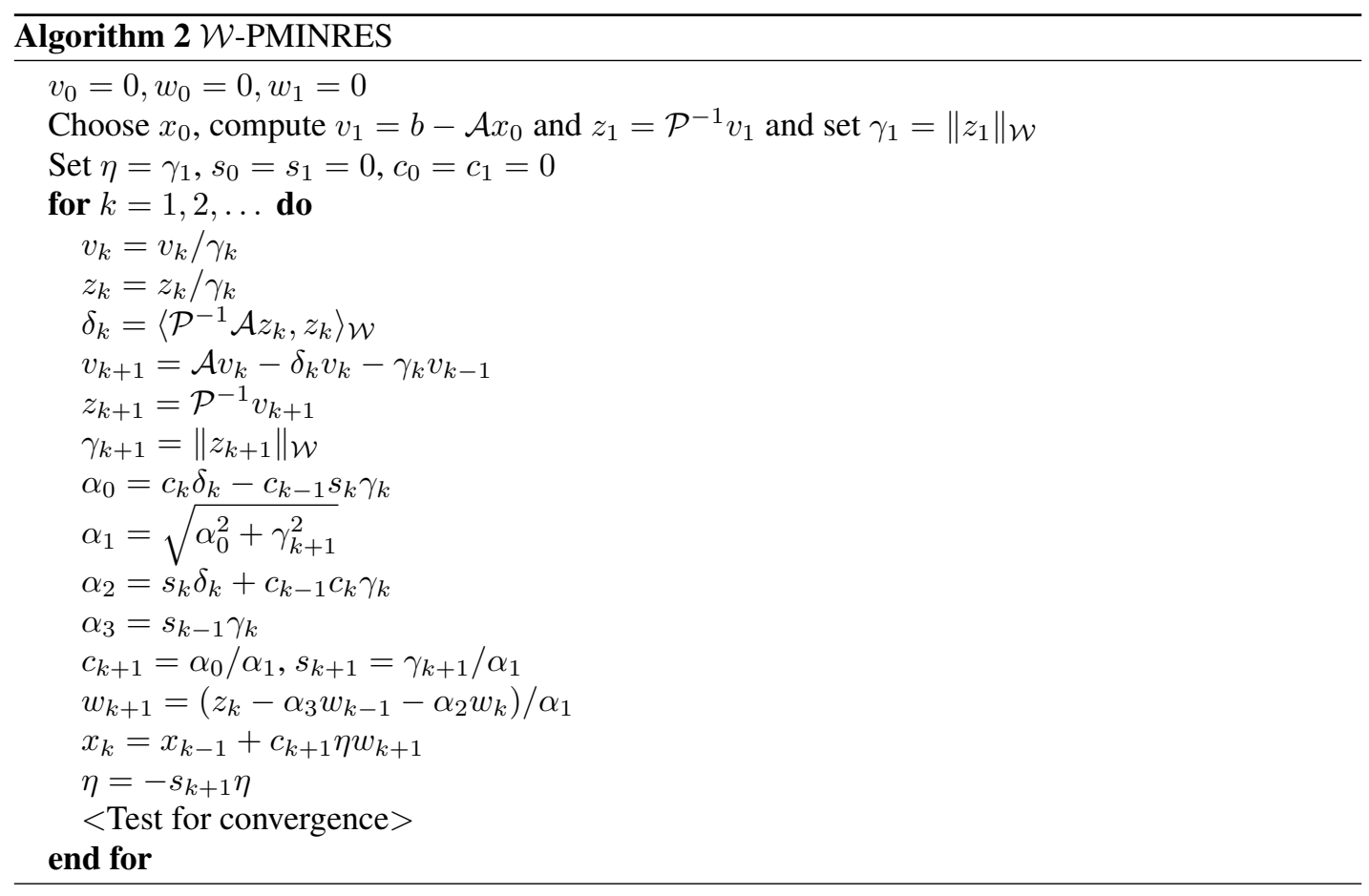

\section{PRECONDITIONERS FOR SADDLE POINT MATRICES}

One of the best known Krylov subspace methods for (1) in a nonstandard inner product is the Bramble-Pasciak (BP) preconditioned conjugate gradient method $[?, ?, ?, ?, ?, ?, ?]$ in which (1) is left-preconditioned by

$$
\mathcal{P}=\left[\begin{array}{cc}
A_{0} & 0 \\
B & -I
\end{array}\right]
$$

where $A_{0}$ is a symmetric approximation to $A$. The coefficient matrix $\mathcal{P}^{-1} \mathcal{A}$ is self-adjoint with respect to $\langle\cdot, \cdot\rangle_{\mathcal{W}}$, where

$$
\mathcal{W}=\left[\begin{array}{cc}
A-A_{0} & 0 \\
0 & I
\end{array}\right]
$$

An inner product is defined by $\mathcal{W}$ when $A-A_{0}>0$ which is also the condition for $\mathcal{P}^{-1} \mathcal{A}$ to be $\mathcal{W}$ positive definite [?]. In this case a $\mathcal{W}$-PCG method, that can be efficiently implemented [?, ?], can be reliably used to solve (1). This coincidence of desirable properties makes the Bramble-Pasciak approach popular.

An alternative is to apply a constraint preconditioner, such as the Schöberl-Zulehner (SZ) preconditioner [?, ?]

$$
\mathcal{P}=\left[\begin{array}{cc}
I & 0 \\
B A_{0}^{-1} & I
\end{array}\right]\left[\begin{array}{cc}
A_{0} & 0 \\
0 & -S_{0}
\end{array}\right]\left[\begin{array}{cc}
I & A_{0}^{-1} B^{T} \\
0 & I
\end{array}\right] .
$$

It is suitable for symmetric saddle point matrices with zero $(2,2)$ block, particularly those arising from optimal control problems $[?, ?, ?]$. The preconditioned saddle point matrix is self-adjoint with respect to the symmetric bilinear form defined by

$$
\mathcal{W}=\left[\begin{array}{cc}
A_{0}-A & 0 \\
0 & B A_{0}^{-1} B^{T}-S_{0}
\end{array}\right]
$$

which is positive definite when $A_{0}>A$ and $B A_{0}^{-1} B^{T}>S_{0}$. Furthermore, when $\langle\cdot, \cdot\rangle_{\mathcal{W}}$ is an inner product, $\mathcal{P}^{-1} \mathcal{A}$ is $\mathcal{W}$-positive definite [?]. 
The major drawback of both the BP and SZ preconditioners is that often $A_{0}$ must be scaled for $\mathcal{W}$ to define an inner product. This typically requires an approximation to the smallest eigenvalue of $A_{0}^{-1} A$, the computation of which can be costly. To circumvent this difficulty, Stoll and Wathen [?] proposed the Bramble-Pasciak ${ }^{+}\left(\mathrm{BP}^{+}\right)$preconditioner

$$
\mathcal{P}=\left[\begin{array}{cc}
A_{0} & 0 \\
-B & S_{0}
\end{array}\right]
$$

for which $\mathcal{P}^{-1} \mathcal{A}$ is self-adjoint with respect to $\langle\cdot, \cdot\rangle_{\mathcal{W}}$, where

$$
\mathcal{W}=\left[\begin{array}{cc}
A+A_{0} & 0 \\
0 & S_{0}
\end{array}\right]
$$

When $A, A_{0}$ and $S_{0}$ are symmetric positive definite, $\mathcal{W}$ is symmetric positive definite and defines an inner product. However, in contrast to the Bramble-Pasciak preconditioners above, $\mathcal{P}^{-1} \mathcal{A}$ is indefinite with respect to $\langle\cdot, \cdot\rangle_{\mathcal{W}}$ [?, Section 5]. Consequently, $\mathcal{W}$-PCG cannot be reliably applied although it is always possible to use $\mathcal{W}$-PMINRES.

Similarly to the $\mathrm{BP}^{+}$preconditioner, the $\mathrm{SZ}$ preconditioner can be modified so that $\mathcal{W}$ always defines an inner product, so that the potentially costly scaling of $A_{0}$ is not required. We call this the Schöberl-Zulehner ${ }^{+}\left(\mathrm{SZ}^{+}\right)$preconditioner. It appeared in [?, Table 3] and is given by

$$
\mathcal{P}=\left[\begin{array}{cc}
I & 0 \\
-B A_{0}^{-1} & I
\end{array}\right]\left[\begin{array}{cc}
A_{0} & 0 \\
0 & S_{0}
\end{array}\right]\left[\begin{array}{cc}
I & -A_{0}^{-1} B^{T} \\
0 & I
\end{array}\right]
$$

so that $\mathcal{P}^{-1} \mathcal{A}$ is self-adjoint with respect to $\langle\cdot, \cdot\rangle_{\mathcal{W}}$, where

$$
\mathcal{W}=\left[\begin{array}{cc}
A+A_{0} & \\
& B A_{0}^{-1} B^{T}+S_{0}
\end{array}\right] .
$$

$\mathcal{W}$ in (13) is clearly positive definite whenever $A+A_{0}>0$ and $B A_{0}^{-1} B^{T}+S_{0}>0$, so that a sufficient condition for positive definiteness is that $A_{0}$ and $S_{0}$ are themselves positive definite, and it is always possible to apply a $\mathcal{W}$-PMINRES algorithm to the $\mathrm{SZ}^{+}$-preconditioned system. However, as we prove below, $\mathcal{P}^{-1} \mathcal{A}$ is never positive definite with respect to this inner product.

\section{Theorem 3}

Let $\mathcal{A}$ in (1), with $C=0$, be left preconditioned by the $\mathrm{SZ}^{+}$preconditioner (12). Then $\mathcal{P}^{-1} \mathcal{A}$ is indefinite with respect to $\langle\cdot, \cdot\rangle_{\mathcal{W}}$, where $\mathcal{W}$ is defined by (13).

\section{Proof}

To ascertain whether $\mathcal{P}^{-1} \mathcal{A}$ is $\mathcal{W}$-positive definite, recall from (4) that an equivalent condition for positive definiteness is that $\mathcal{W} \mathcal{P}^{-1} \mathcal{A}$ be positive definite with respect to the Euclidean inner product. Accordingly, we first factor $\mathcal{W} \mathcal{P}^{-1} \mathcal{A}$ as

$$
\begin{aligned}
\mathcal{W P}^{-1} \mathcal{A} & =\left[\begin{array}{cc}
A+A_{0} & \\
& B A_{0}^{-1} B^{T}+S_{0}
\end{array}\right]\left[\begin{array}{cc}
A_{0}^{-1} & A_{0}^{-1} B^{T} S_{0}^{-1} \\
& S_{0}^{-1}
\end{array}\right]\left[\begin{array}{cc}
I \\
B A_{0}^{-1} & I
\end{array}\right]\left[\begin{array}{cc}
A & B^{T} \\
B & 0
\end{array}\right] \\
& =\left[\begin{array}{cc}
I & \\
B W\left(A+A_{0}\right) M^{-1} & I
\end{array}\right]\left[\begin{array}{cc}
M & \\
& T
\end{array}\right]\left[\begin{array}{cc}
I & M^{-1}\left(A+A_{0}\right) W B^{T} \\
I
\end{array}\right],
\end{aligned}
$$

where

$$
\begin{gathered}
M=A+A A_{0}^{-1} A+\left(A+A_{0}\right) A_{0}^{-1} B^{T} S_{0}^{-1} B A_{0}^{-1}\left(A+A_{0}\right), \\
W=A_{0}^{-1}+A_{0}^{-1} B^{T} S_{0}^{-1} B A_{0}^{-1}
\end{gathered}
$$

and

$$
T=B W\left(W^{-1}-\left(A+A_{0}\right) M^{-1}\left(A+A_{0}\right)\right) W B^{T} .
$$


The congruence transform (14) shows that $\mathcal{P}^{-1} \mathcal{A}$ is positive definite with respect to $\langle\cdot, \cdot\rangle_{\mathcal{W}}$ whenever $M>0$ and $T>0$, i.e., whenever $M$ and $T$ are positive definite. Since $A_{0}$ and $S_{0}$ are positive definite and $B$ has full rank, $W$ and $M$, given by (15) and (16), are the sum of positive definite matrices and so are positive definite. However, the matrix $T$ in (17) is negative definite as we now show. Negative definiteness of $T$ is equivalent to

$$
W^{-1}<\left(A+A_{0}\right) M^{-1}\left(A+A_{0}\right)
$$

(where $>$ represents the positive semi-definite ordering) or, since $M$ and $W$ are positive definite, to

$$
W>\left(A+A_{0}\right)^{-1} M\left(A+A_{0}\right)^{-1} .
$$

Substituting for $W$ and $M$ gives that

$$
A_{0}^{-1}+A_{0}^{-1} B^{T} S_{0}^{-1} B A_{0}^{-1}>A_{0}^{-1} A\left(A+A_{0}\right)^{-1}+A_{0}^{-1} B^{T} S_{0}^{-1} B A_{0}^{-1}
$$

or that

$$
0>A_{0}^{-1} A\left(A+A_{0}\right)^{-1}-A_{0}^{-1} .
$$

Now,

$$
A_{0}^{-1} A\left(A+A_{0}\right)^{-1}=A_{0}^{-1}\left[A\left(A+A_{0}\right)^{-1} A_{0}\right] A_{0}^{-1}=A_{0}^{-1}\left(A^{-1}+A_{0}^{-1}\right)^{-1} A_{0}^{-1}
$$

and so (18) can be expressed as

$$
0>A_{0}^{-1}\left(\left(A^{-1}+A_{0}^{-1}\right)^{-1}-A_{0}\right) A_{0}^{-1} .
$$

This holds if and only if

$$
A_{0}^{-1}<A_{0}^{-1}+A^{-1}
$$

which, since $A^{-1}$ is positive definite, is satisfied. Therefore, $T$ is negative definite while $M$ is positive definite. By considering (14) we see that by Sylvester's law of inertia $\mathcal{W P}^{-1} \mathcal{A}$ is indefinite. It follows from (4) that $\mathcal{P}^{-1} \mathcal{A}$ is indefinite with respect to $\langle\cdot, \cdot\rangle_{\mathcal{W}}$.

A third preconditioner for which $\mathcal{P}^{-1} \mathcal{A}$ is always indefinite with respect to an inner product is the block diagonal $(\mathrm{BD})$ preconditioner $[?, ?, ?]$

$$
\mathcal{P}=\left[\begin{array}{cc}
A_{0} & 0 \\
0 & S_{0}
\end{array}\right]
$$

for which $\mathcal{P}^{-1} \mathcal{A}$ is self-adjoint with respect to the symmetric bilinear form defined by

$$
\mathcal{W}=\left[\begin{array}{cc}
A_{0} & 0 \\
0 & S_{0}
\end{array}\right]
$$

Indeed, the self-adjointness requirement (3) that $\mathcal{W P}^{-1} \mathcal{A}$ is symmetric is trivially satisfied since $\mathcal{W} \mathcal{P}^{-1} \mathcal{A}=\mathcal{A}$, the original symmetric saddle point matrix. The indefiniteness of $\mathcal{A}$, however, means that $\mathcal{P}^{-1} \mathcal{A}$ is indefinite with respect to $\langle\cdot, \cdot\rangle_{\mathcal{W}}$.

Each of the preconditioners in this section is a special instance of the Krzyżanowski preconditioner [?]

$$
\mathcal{P}=\left[\begin{array}{cc}
I & 0 \\
c B A_{0}^{-1} & I
\end{array}\right]\left[\begin{array}{cc}
A_{0} & 0 \\
0 & S_{0}
\end{array}\right]\left[\begin{array}{cc}
I & d A_{0}^{-1} B^{T} \\
0 & I
\end{array}\right],
$$

with $|c|,|d| \leq 1$. The specific parameter values are given in Table I. As shown in [?], the Krzyżanowski preconditioned matrix $\mathcal{P}^{-1} \mathcal{A}$ is self-adjoint with respect to $\langle\cdot, \cdot\rangle_{\mathcal{W}}$, where

$$
\mathcal{W}=\epsilon\left[\begin{array}{cc}
A_{0}-c A & 0 \\
0 & S_{0}+c d B A_{0}^{-1} B^{T}+d C
\end{array}\right]
$$


and $\epsilon= \pm 1$.

It appears that the $\mathcal{W}$-PCG method in Algorithm 1 and the $\mathcal{W}$-PMINRES method in Algorithm 2 require $A_{0}$ and $S_{0}$ (for inner products with $\mathcal{W}$ ) and their inverses (to apply $\mathcal{P}^{-1}$ to vectors). However, as shown by Krzyżanowski [?], this is not the case. To see this, consider the computation of the inner product $\left\langle z_{k}, z_{k}\right\rangle_{\mathcal{W}}$ in Algorithm 1. Since

$$
\left\langle z_{k}, z_{k}\right\rangle_{\mathcal{W}}=z_{k}^{T} \mathcal{W} \mathcal{P}^{-1} r_{k}
$$

and [?, Section 2]

$$
\mathcal{W P}^{-1}=\epsilon\left[\begin{array}{cc}
I & 0 \\
0 & I
\end{array}\right]-\epsilon \mathcal{A}\left[\begin{array}{cc}
c I & 0 \\
0 & d I
\end{array}\right] \mathcal{P}^{-1},
$$

only the actions of $A_{0}^{-1}$ and $S_{0}^{-1}$ on vectors are required, as well as multiplications with $A, B$ and $C$. Other inner products can be treated similarly.

The Krzyżanowski preconditioner allows us to make statements that apply to each of the previously described preconditioners, along with several others [?]. Lemmas 1 and 2 indicate that eigenvalues influence the convergence of a $\mathcal{W}$-PCG or $\mathcal{W}$-PMINRES method applied to the Krzyżanowski preconditioned system. We prove below that the eigenvalues of the Krzyżanowski preconditioned saddle point matrix depend on certain generalized Rayleigh quotients when $C=0$.

Lemma 4

Let the symmetric saddle point matrix

$$
\mathcal{A}=\left[\begin{array}{cc}
A & B^{T} \\
B & 0
\end{array}\right]
$$

be left preconditioned by the Krzyżanowski preconditioner (21) and let $\mathcal{W}$ in (22) be positive definite. If $\lambda$ is an eigenvalue of $\mathcal{P}^{-1} \mathcal{A}$ with associated eigenvector

$$
\left[\begin{array}{ll}
u_{1}^{T}, & u_{2}^{T}
\end{array}\right]^{T},
$$

$u_{1} \in \mathbb{R}^{n}, u_{2} \in \mathbb{R}^{m}$, then $\lambda$ is real. Additionally, $\lambda \neq 0$ and when $c$ is nonzero $\lambda \neq 1 / c$. If $B u_{1}=0$ then

$$
\lambda=\frac{u_{1}^{T} A u_{1}}{u_{1}^{T} A_{0} u_{1}} .
$$

Otherwise,

$$
\lambda=\frac{1-(c+d) \omega \pm \sqrt{(1-(c+d) \omega)^{2}+4(\psi-c d \omega) \omega}}{2(\psi-c d \omega)}
$$

where

$$
\psi=\frac{u_{1}^{T} A_{0} u_{1}}{u_{1}^{T} A u_{1}}
$$

and

$$
\omega=\frac{u_{1}^{T} B^{T}\left(c d B A_{0}^{-1} B^{T}+S_{0}\right)^{-1} B u_{1}}{u_{1}^{T} A u_{1}} .
$$

Proof

The eigenvalues of $\mathcal{P}^{-1} \mathcal{A}$ are those of the generalized eigenvalue problem

$$
\left[\begin{array}{cc}
A & B^{T} \\
B & 0
\end{array}\right]\left[\begin{array}{l}
u_{1} \\
u_{2}
\end{array}\right]=\lambda\left[\begin{array}{cc}
A_{0} & d B^{T} \\
c B & c d B A_{0}^{-1} B^{T}+S_{0}
\end{array}\right]\left[\begin{array}{l}
u_{1} \\
u_{2}
\end{array}\right]
$$

which, in component form, is

$$
\begin{gathered}
A u_{1}+B^{T} v=\lambda A_{0} u_{1}+\lambda d B^{T} u_{2} \\
B u_{1}=\lambda c B u_{1}+\lambda\left(c d B A_{0}^{-1} B^{T}+S_{0}\right) u_{2} .
\end{gathered}
$$


Since $\mathcal{P}^{-1} \mathcal{A}$ is self-adjoint with respect to an inner product its eigenvalues are real. If $\lambda=0$, (28) implies that $B u_{1}=0$. Premultiplying (27) by $u_{1}^{T}$ gives that $u_{1}^{T} A u_{1}=0$ which, since $A$ is positive definite, can only be true if $u_{1}=0$. However, then (27) reduces to $B^{T} u_{2}=0$. The matrix $B$ has full rank and it follows that $u_{2}=0$. We conclude that zero is not an eigenvalue of $\mathcal{P}^{-1} \mathcal{A}$. More generally, $u_{1} \neq 0$ since the definiteness of $S_{0}+c d B A_{0}^{-1} B^{T}$ - which follows from the assumption of positive definiteness of $\mathcal{W}$-means that when $u_{1}=0,(28)$ can only be satisfied when $u_{2}=0$.

If $c \neq 0$ and $\lambda=1 / c$ we again find from (28) that $u_{2}=0$. It follows that (27) can be simplified and rearranged to give that $\left(A_{0}-c A\right) u_{1}=0$. However, positive definiteness of $\mathcal{W}$ ensures that $A_{0}-c A$ is definite which shows that $u_{1}=0$. Thus, $\lambda \neq 1 / c$.

Let us now determine the eigenvalues of $\mathcal{P}^{-1} \mathcal{A}$. When $B u_{1}=0$, (28) becomes

$$
\lambda\left(c d B A_{0}^{-1} B^{T}+S_{0}\right) u_{2}=0
$$

which implies that $v=0$, since $c d B A_{0}^{-1} B^{T}+S_{0}$ is definite and $\lambda \neq 0$. Substituting $u_{2}=0$ into (27) gives that $A u_{1}=\lambda A_{0} u_{1}$ which, since $u_{1} \neq 0$, yields that

$$
\lambda=\frac{u_{1}^{T} A u_{1}}{u_{1}^{T} A_{0} u_{1}} .
$$

When $B u_{1} \neq 0$, rearranging (28) gives that

$$
u_{2}=\left(\frac{1}{\lambda}-c\right) \tilde{S}^{-1} B u_{1}
$$

where $\tilde{S}=c d B A_{0}^{-1} B^{T}+S_{0}$. By substituting for $u_{2}$ in (27) we obtain the equation

$$
A u_{1}+(1-\lambda d)\left(\frac{1}{\lambda}-c\right) B^{T} \tilde{S}^{-1} B u_{1}=\lambda A_{0} u_{1}
$$

for $\lambda$. Premultiplying by $\lambda u_{1}^{T}$ and dividing by $u_{1}^{T} A u_{1}$ leads to a quadratic equation with roots given by

$$
\lambda=\frac{1-(c+d) \omega \pm \sqrt{(1-(c+d) \omega)^{2}+4(\psi-c d \omega) \omega}}{2(\psi-c d \omega)} .
$$

Remark 1. Although (24) is complicated it simplifies in each of the cases in Table I and so provides useful information in these specific instances. Knowledge of the extreme values of (25) and (26) allow the eigenvalues of $\mathcal{P}^{-1} \mathcal{A}$ to be bounded and a priori information about the convergence of a $\mathcal{W}$-Krylov subspace method to be obtained.

\section{COMBINATION PRECONDITIONING}

Combination preconditioning [?] allows two preconditioners, for each of which the preconditioned coefficient matrix is self-adjoint with respect to a symmetric bilinear form, to be blended. The result is a new preconditioner and a symmetric bilinear form with respect to which the combination preconditioned coefficient matrix is self-adjoint. The process is controlled by two parameters, for certain choices of which the combination preconditioner is more effective than either of the original preconditioners and is no more costly to apply than the more expensive of the two. The process of combination preconditioning is described in Lemma 3.5 in [?] which we reproduce below using our notation. 
Lemma 5

If $\mathcal{P}_{1}$ and $\mathcal{P}_{2}$ are left preconditioners for the symmetric matrix $\mathcal{A}$ for which symmetric matrices $\mathcal{W}_{1}$ and $\mathcal{W}_{2}$ exist with $\mathcal{P}_{1}^{-1} \mathcal{A}$ self-adjoint in $\langle\cdot, \cdot\rangle_{\mathcal{W}_{1}}$ and $\mathcal{P}_{2}^{-1} \mathcal{A}$ self-adjoint in $\langle\cdot, \cdot\rangle_{\mathcal{W}_{2}}$ and if

$$
\alpha \mathcal{P}_{1}^{-T} \mathcal{W}_{1}+\beta \mathcal{P}_{2}^{-T} \mathcal{W}_{2}=\mathcal{P}_{3}^{-T} \mathcal{W}_{3}
$$

for some matrix $\mathcal{P}_{3}$ and some symmetric matrix $\mathcal{W}_{3}$, then $\mathcal{P}_{3}^{-1} \mathcal{A}$ is self-adjoint in $\langle\cdot, \cdot\rangle_{\mathcal{W}_{3}}$.

To combine preconditioners described in the previous section, we first apply Lemma 5 to two different Krzyżanowski preconditioners

$$
\mathcal{P}_{1}=\left[\begin{array}{cc}
I & 0 \\
c_{1} B A_{0}^{-1} & I
\end{array}\right]\left[\begin{array}{cc}
A_{0} & 0 \\
0 & S_{0}
\end{array}\right]\left[\begin{array}{cc}
I & d_{1} A_{0}^{-1} B^{T} \\
0 & I
\end{array}\right],
$$

and

$$
\mathcal{P}_{2}=\left[\begin{array}{cc}
I & 0 \\
c_{2} B A_{0}^{-1} & I
\end{array}\right]\left[\begin{array}{cc}
A_{0} & 0 \\
0 & S_{0}
\end{array}\right]\left[\begin{array}{cc}
I & d_{2} A_{0}^{-1} B^{T} \\
0 & I
\end{array}\right]
$$

with bilinear forms defined by

$$
\mathcal{W}_{1}=\epsilon_{1}\left[\begin{array}{cc}
A_{0}-c_{1} A & 0 \\
0 & S_{0}+c_{1} d_{1} B A_{0}^{-1} B^{T}+d_{1} C
\end{array}\right]
$$

and

$$
\mathcal{W}_{2}=\epsilon_{2}\left[\begin{array}{cc}
A_{0}-c_{2} A & 0 \\
0 & S_{0}+c_{2} d_{2} B A_{0}^{-1} B^{T}+d_{2} C
\end{array}\right] .
$$

The following theorem shows that under certain conditions a combination preconditioner can be constructed that retains the structure of a Krzyżanowski preconditioner.

\section{Theorem 6}

Let $\mathcal{P}_{1}, \mathcal{P}_{2}, \mathcal{W}_{1}$ and $\mathcal{W}_{2}$ be defined by (29), (30), (31) and (32).Then, if $c_{1}=c_{2}=c$,

$$
\mathcal{P}=\mathcal{P}_{3}=\left[\begin{array}{cc}
I & 0 \\
c B A_{0}^{-1} & I
\end{array}\right]\left[\begin{array}{cc}
\frac{1}{\alpha \epsilon_{1}+\beta \epsilon_{2}} A_{0} & 0 \\
0 & S_{0}
\end{array}\right]\left[\begin{array}{cc}
I & \left(\alpha \epsilon_{1} d_{1}+\beta \epsilon_{2} d_{2}\right) A_{0}^{-1} B^{T} \\
0 & I
\end{array}\right]
$$

is a combination preconditioner, formed from $\mathcal{P}_{1}$ and $\mathcal{P}_{2}$, for which $\mathcal{P}^{-1} \mathcal{A}$ is self-adjoint with respect to the symmetric bilinear form defined by

$$
\mathcal{W}=\mathcal{W}_{3}=\left[\begin{array}{cc}
\left(A_{0}-c A\right) & 0 \\
0 & \left(\alpha \epsilon_{1}+\beta \epsilon_{2}\right) S_{0}+\left(\alpha \epsilon_{1} d_{1}+\beta \epsilon_{2} d_{2}\right)\left(c B A_{0}^{-1} B^{T}+C\right)
\end{array}\right]
$$

Alternatively, if $d_{1}=d_{2}=0$, the combination preconditioner

$$
\mathcal{P}=\mathcal{P}_{3}=\left[\begin{array}{cc}
I & 0 \\
\left(\alpha \epsilon_{1} c_{1}+\beta \epsilon_{2} c_{2}\right) B A_{0}^{-1} & I
\end{array}\right]\left[\begin{array}{cc}
A_{0} & 0 \\
0 & \frac{1}{\alpha \epsilon_{1}+\beta \epsilon_{2}} S_{0}
\end{array}\right]
$$

is such that $\mathcal{P}^{-1} \mathcal{A}$ is self-adjoint with respect to the symmetric bilinear form defined by

$$
\mathcal{W}=\mathcal{W}_{3}=\left[\begin{array}{cc}
\left(\alpha \epsilon_{1}+\beta \epsilon_{2}\right) A_{0}-\left(\alpha \epsilon_{1} c_{1}+\beta \epsilon_{3} c_{2}\right) A & 0 \\
0 & S_{0}
\end{array}\right]
$$

Proof

In general, application of Lemma 5 to (29), (30), (31) and (32) gives that

$$
\begin{aligned}
\mathcal{P}_{3}^{-T} \mathcal{W}_{3} & =\alpha \mathcal{P}_{1}^{-T} \mathcal{W}_{1}+\beta \mathcal{P}_{2}^{-T} \mathcal{W}_{2} \\
& =\alpha \epsilon_{1}\left[\begin{array}{ll}
G_{11}^{(1)} & G_{12}^{(1)} \\
G_{21}^{(1)} & G_{22}^{(1)}
\end{array}\right]+\beta \epsilon_{2}\left[\begin{array}{ll}
G_{11}^{(2)} & G_{12}^{(2)} \\
G_{21}^{(2)} & G_{22}^{(2)}
\end{array}\right]
\end{aligned}
$$


where, for $i=1,2$,

$$
\begin{aligned}
G_{11}^{(i)} & =\left(A_{0}^{-1}+c_{i} d_{i} A_{0}^{-1} B^{T} S_{0}^{-1} B A_{0}^{-1}\right)\left(A_{0}-c_{i} A\right), \\
G_{12}^{(i)} & =-c_{i} A_{0}^{-1} B^{T} S_{0}^{-1}\left(S_{0}+c_{i} d_{i} B A_{0}^{-1} B^{T}+d_{i} C\right), \\
G_{21}^{(i)} & =-d_{i} S_{0}^{-1} B A_{0}^{-1}\left(A_{0}-c_{i} A\right), \\
G_{22}^{(i)} & =S_{0}^{-1}\left(S_{0}+c_{i} d_{i} B A_{0}^{-1} B^{T}+d_{i} C\right) .
\end{aligned}
$$

When $c_{1}=c_{2}=c$, it is straightforward to show that (33) becomes

$$
\mathcal{P}_{3}^{-T} \mathcal{W}=\left[\begin{array}{ll}
P_{11} W_{11} & P_{12} W_{22} \\
P_{21} W_{11} & P_{22} W_{22}
\end{array}\right]
$$

where

$$
\begin{aligned}
& P_{11}=\left(\alpha \epsilon_{1}+\beta \epsilon_{2}\right) A_{0}^{-1}+\left(\alpha \epsilon_{1} d_{1}+\beta \epsilon_{2} d_{2}\right) c A_{0}^{-1} B^{T} S_{0}^{-1} B A_{0}^{-1}, \\
& P_{12}=-c A_{0}^{-1} B^{T} S_{0}^{-1}, \\
& P_{21}=-\left(\alpha \epsilon_{1} d_{1}+\beta \epsilon_{2} d_{2}\right) S_{0}^{-1} B A_{0}^{-1}\left(A_{0}-c A\right), \\
& P_{22}=S_{0}^{-1}
\end{aligned}
$$

$W_{11}=A_{0}-c A$ and $W_{22}=\left(\alpha \epsilon_{1}+\beta \epsilon_{2}\right) S_{0}+\left(\alpha \epsilon_{1} d_{1}+\beta \epsilon_{2} d_{2}\right)\left(c B A_{0}^{-1} B^{T}+C\right)$. Thus, a natural choice is

$$
\mathcal{P}_{3}^{-1}=\left[\begin{array}{ll}
P_{11}^{T} & P_{21}^{T} \\
P_{12}^{T} & P_{22}^{T}
\end{array}\right]
$$

so that

$$
\mathcal{W}_{3}=\left[\begin{array}{cc}
W_{11} & 0 \\
0 & W_{22}
\end{array}\right]
$$

and $\mathcal{P}_{3}$ is as stated in the theorem.

Similarly, when $d_{1}=d_{2}=0$ we find from (33) and (34) that

$$
\mathcal{P}_{3}^{-T} \mathcal{W}_{3}=\left[\begin{array}{cc}
A_{0}^{-1}\left(\left(\alpha \epsilon_{1}+\beta \epsilon_{2}\right) A_{0}+\left(\alpha \epsilon_{1} c_{1}+\beta \epsilon_{2} c_{2} A\right)\right) & -\left(\alpha \epsilon_{1} c_{1}+\beta \epsilon_{2} c_{2}\right) A_{0}^{-1} B^{T} S_{0}^{-1} \\
0 & I
\end{array}\right]
$$

and so we can take

$$
\mathcal{P}_{3}^{-1}=\left[\begin{array}{cc}
A_{0}^{-1} & 0 \\
0 & \left(\alpha \epsilon_{1}+\beta \epsilon_{2}\right) S_{0}^{-1}
\end{array}\right]\left[\begin{array}{cc}
I & 0 \\
-\left(\alpha \epsilon_{1} c_{1}+\beta \epsilon_{2} c_{2}\right) B A_{0}^{-1} & I
\end{array}\right]
$$

and

$$
\mathcal{W}_{3}=\left[\begin{array}{cc}
\left(\alpha \epsilon_{1}+\beta \epsilon_{2}\right) A_{0}-\left(\alpha \epsilon_{1} c_{1}+\beta \epsilon_{3} c_{2}\right) A & 0 \\
0 & S_{0}
\end{array}\right]
$$

Stoll and Wathen [?] introduced a BP-BP ${ }^{+}$combination preconditioner and a BP-SZ combination preconditioner, the former of which takes $\beta=1-\alpha$ and the latter of which differs from the preconditioner that would be obtained by Theorem 6. Clearly, combination preconditioners are not uniquely defined, although the above characterization certainly makes it straightforward to construct combinations of preconditioners that satisfy the conditions of the theorem. 


\subsection{The $\mathrm{BP}^{+}-\mathrm{SZ}^{+}$combination preconditioner}

Theorem 6 applied to the $\mathrm{BP}^{+}$preconditioner (11) and $\mathrm{SZ}^{+}$preconditioner (12), for which $c_{1}=$ $c_{2}=-1, d_{1}=0, d_{2}=-1$ and $\epsilon_{1}=\epsilon_{2}=1$, gives that

$$
\mathcal{P}=\left[\begin{array}{cc}
I & 0 \\
-B A_{0}^{-1} & I
\end{array}\right]\left[\begin{array}{cc}
\frac{1}{\alpha+\beta} A_{0} & \frac{-\beta}{\alpha+\beta} B^{T} \\
0 & S_{0}
\end{array}\right]
$$

and

$$
\mathcal{W}=\mathcal{W}_{3}=\left[\begin{array}{cc}
A+A_{0} & 0 \\
0 & (\alpha+\beta) S_{0}+\beta B A_{0}^{-1} B^{T}
\end{array}\right] .
$$

However, like the $\mathrm{BP}^{+}$and $\mathrm{SZ}^{+}$preconditioners, the combination is never positive definite with respect to an inner product.

\section{Theorem 7}

Let

$$
\mathcal{A}=\left[\begin{array}{cc}
A & B^{T} \\
B & 0
\end{array}\right]
$$

be left preconditioned by the $\mathrm{BP}^{+}-\mathrm{SZ}^{+}$combination preconditioner (36), so that $\mathcal{P}^{-1} \mathcal{A}$ is selfadjoint with respect to $\langle\cdot, \cdot\rangle_{\mathcal{W}}$, where $\mathcal{W}$ is defined by (37).

When

I. $\alpha+\beta>0$ and $\beta>0, \mathcal{W}$ defines an inner product;

II. $\alpha+\beta<0$ and $\beta>0, \mathcal{W}$ defines an inner product when

$$
S_{0}<-\frac{\beta}{\alpha+\beta} B A_{0}^{-1} B^{T}
$$

III. $\alpha+\beta<0$ and $\beta<0 \mathcal{W}$ does not define an inner product;

IV. $\alpha+\beta>0$ and $\beta<0, \mathcal{W}$ defines an inner product when

$$
S_{0}>-\frac{\beta}{\alpha+\beta} B A_{0}^{-1} B^{T}
$$

When $\langle\cdot, \cdot\rangle_{\mathcal{W}}$ is an inner product, it is one with respect to which $\mathcal{P}^{-1} \mathcal{A}$ is not positive definite.

Proof

By (4), $\mathcal{P}^{-1} \mathcal{A}$ is $\mathcal{W}$-positive definite if and only if $\mathcal{W} \mathcal{P}^{-1} \mathcal{A}>0$, where

$$
\mathcal{W P}^{-1} \mathcal{A}=\left[\begin{array}{cc}
I & 0 \\
B V\left(A+A_{0}\right) M^{-1} & I
\end{array}\right]\left[\begin{array}{cc}
M & 0 \\
0 & T
\end{array}\right]\left[\begin{array}{cc}
I & M^{-1}\left(A+A_{0}\right) V B^{T} \\
0 & I
\end{array}\right],
$$

with

$$
\begin{gathered}
M=(\alpha+\beta)\left(A+A_{0}\right) A_{0}^{-1} A+\beta\left(A+A_{0}\right) A_{0}^{-1} B^{T} S_{0}^{-1} B A_{0}^{-1}\left(A+A_{0}\right) \\
=(\alpha+\beta) A\left(A^{-1}+A_{0}^{-1}\right) A+\beta\left(A+A_{0}\right) A_{0}^{-1} B^{T} S_{0}^{-1} B A_{0}^{-1}\left(A+A_{0}\right), \\
T=B V\left[V^{-1}-\left(A+A_{0}\right) M^{-1}\left(A+A_{0}\right)\right] V B^{T} .
\end{gathered}
$$

and

$$
V=(\alpha+\beta) A_{0}^{-1}+\beta A_{0}^{-1} B^{T} S_{0}^{-1} B A_{0}^{-1}
$$




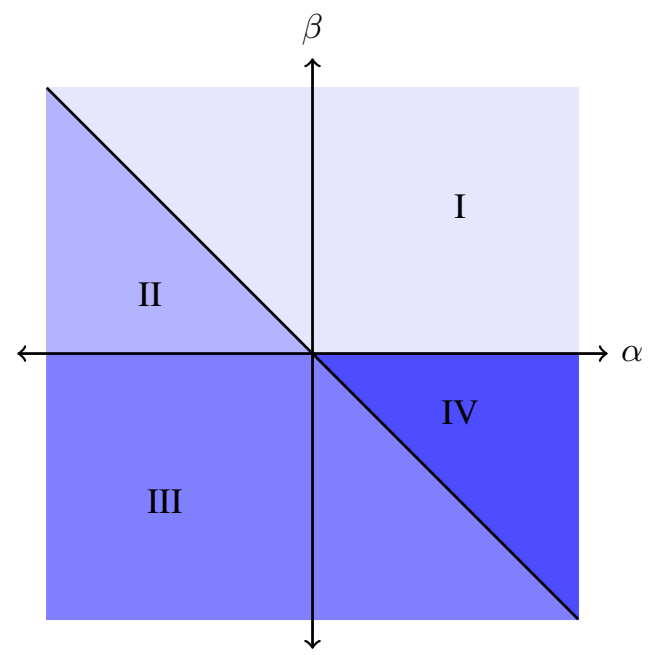

Figure 1. Regions (distinguished by different colours) that are considered in Theorem 7 for the $\mathrm{BP}^{+}-\mathrm{SZ}^{+}$ combination preconditioner.

The congruence transform (38) shows that the inertia of $\mathcal{W} \mathcal{P}^{-1} \mathcal{A}$ is determined by the eigenvalues of $M$ and $T$ which, in turn, depend on $\alpha$ and $\beta$. We consider separately each of the four cases in Figure 1.

I. Case I corresponds to taking $\alpha+\beta>0$ with $\beta>0$. By observation, $\mathcal{W}, V$ and $M$ are positive definite. However, $T$ in (40) is negative definite. To show this, we first observe that $T<0$ if and only if

$$
V^{-1}<\left(A+A_{0}\right) M^{-1}\left(A+A_{0}\right)
$$

or, since $V$ and $M$ are positive definite,

$$
V>\left(A+A_{0}\right)^{-1} M\left(A+A_{0}\right)^{-1} .
$$

It follows from substituting for $V$ and $M$ using (41) and (39), that $T$ is negative definite if and only if

$$
(\alpha+\beta) A_{0}^{-1}>(\alpha+\beta) A_{0}^{-1} A\left(A+A_{0}\right)^{-1} .
$$

Premultiplying and postmultiplying both sides of this inequality by $A_{0}$ and dividing by the positive scalar $\alpha+\beta$ gives the equivalent condition

$$
A_{0}>A\left(A+A_{0}^{-1}\right)^{-1} A_{0}=\left(A_{0}^{-1}+A^{-1}\right)^{-1}
$$

which implies that $A_{0}^{-1}<A_{0}^{-1}+A^{-1}$. Since $A>0$, this last inequality holds and $T$ is negative definite. We conclude that $\mathcal{P}^{-1} \mathcal{A}$ is indefinite with respect to $\langle\cdot, \cdot\rangle_{\mathcal{W}}$, when $\alpha+\beta>0$ and $\beta>0$.

II. Consider now $\alpha+\beta<0$ and $\beta>0$ and let $\gamma$ be the positive parameter $\gamma=-\frac{\beta}{\alpha+\beta}$. For these values of $\alpha$ and $\beta,(\alpha+\beta) S_{0}+\beta B A_{0}^{-1} B^{T}>0$ when $\gamma B A_{0}^{-1} B^{T}>S_{0}$.

The matrix $M$ given by (39) is positive definite if and only if

$$
(\alpha+\beta)\left(A+A_{0}\right) A_{0}^{-1} A+\beta\left(A+A_{0}\right) A_{0}^{-1} B^{T} S_{0}^{-1} B A_{0}^{-1}\left(A+A_{0}\right)>0
$$

or, upon premultiplying by $A_{0}\left(A+A_{0}\right)$ and postmultiplying by its transpose, if and only if

$$
(\alpha+\beta) A\left(A+A_{0}\right)^{-1} A_{0}+\beta B^{T} S_{0} B>0
$$

or, equivalently, $\left(A^{-1}-A_{0}^{-1}\right)^{-1}<\gamma B^{T} S_{0}^{-1} B$. However, since $B$ has a nontrivial nullspace $\gamma B^{T} S_{0}^{-1} B$ is positive semidefinite. In contrast, $\left(A_{0}^{-1}+A^{-1}\right)^{-1}$ is positive definite. Consequently, $M$ is not positive definite and $\mathcal{P}^{-1} \mathcal{A}$ is not positive definite with respect to $\langle\cdot, \cdot\rangle_{\mathcal{W}}$. 
III. The third case we investigate is $\alpha+\beta<0$ and $\beta<0$. For these choices of $\alpha$ and $\beta$, however, $(\alpha+\beta) S_{0}+\beta B A_{0}^{-1} B^{T}<0$ and $A+A_{0}>0$ and $\mathcal{W}$ is indefinite.

IV. We consider now Case IV, for which $\alpha+\beta>0$ and $\beta<0$ and introduce the quantity $\gamma=-\frac{\beta}{\alpha+\beta}>0$. Now, $\mathcal{W}>0$ when $(\alpha+\beta) S_{0}+\beta B A_{0}^{-1} B^{T}>0$ or, if $\lambda_{\min }$ and $\lambda_{\max }$ are the smallest and largest eigenvalues of $S_{0}^{-1} B A_{0}^{-1} B^{T}$, respectively, when

$$
\frac{1}{\gamma} \geq \lambda_{\max } \geq \frac{x^{T} B A_{0}^{-1} B^{T} x}{x^{T} S_{0} x} \geq \lambda_{\min }>0 .
$$

Let us express this inequality in a form that is more conducive to determining whether $M$ is positive definite. Any eigenvalue $\lambda$ of $S_{0}^{-1} B A_{0}^{-1} B^{T}$ satisfies the eigenvalue problem

$$
\begin{aligned}
S_{0}^{-1} B A_{0}^{-1} B^{T} x & =\lambda x \\
\Rightarrow A_{0}^{-1} B^{T} S_{0}^{-1} B\left(A_{0}^{-1} B^{T} x\right) & =\lambda A_{0}^{-1} B^{T} x \\
\Rightarrow B^{T} S_{0}^{-1} B y & =A_{0} \lambda y,
\end{aligned}
$$

where $y=A_{0}^{-1} B^{T} x$. (Note that $y=0 \Leftrightarrow x=0$.) Thus, any eigenvalue of $S_{0}^{-1} B A_{0}^{-1} B^{T} \in \mathbb{R}^{m \times m}$ is an eigenvalue of $A_{0}^{-1} B^{T} S_{0}^{-1} B \in \mathbb{R}^{n \times n}$. To determine the remaining $n-m$ eigenvalues of $A_{0}^{-1} B^{T} S_{0}^{-1} B$, we note that this matrix is similar to $A_{0}^{-1 / 2} B^{T} S_{0}^{-1} B A_{0}^{-1 / 2}$ which, in turn, is congruent to the rank $m$ matrix $B^{T} S_{0}^{-1} B \in \mathbb{R}^{n \times n}$. It follows from Sylvester's law of inertia that $A_{0}^{-1} B^{T} S_{0}^{-1} B$, like $B^{T} S_{0}^{-1} B$, has $n-m$ zero eigenvalues.

If $\lambda_{\min }$ and $\lambda_{\max }$ are the minimum and maximum eigenvalues of $S_{0}^{-1} B A_{0}^{-1} B^{T}$ they bound the generalized Rayleigh quotient

$$
\lambda_{\min } \leq \frac{x^{T} B A_{0}^{-1} B^{T} x}{x^{T} S_{0} x} \leq \lambda_{\max }
$$

and it follows that

or that

$$
\frac{1}{\gamma}>\lambda_{\max } \geq \frac{y^{T} B^{T} S_{0}^{-1} B y}{y^{T} A_{0} y} \geq 0
$$

$$
A_{0}^{-1}>\gamma B^{T} S_{0}^{-1} B
$$

is an equivalent condition for positive definiteness of $\mathcal{W}$.

By examining (41) we find that $V>0$ if and only if

$$
(\alpha+\beta) A_{0}^{-1}>-\beta A_{0}^{-1} B^{T} S_{0}^{-1} B A_{0}^{-1}
$$

or, equivalently

$$
A_{0}^{-1}>\gamma B^{T} S_{0}^{-1} B
$$

which is precisely the same as (42) and so is satisfied when $\mathcal{W}$ defines an inner product.

Now, $M>0$ if and only if

$$
(\alpha+\beta)\left(A+A_{0}\right) A_{0}^{-1} A+\beta\left(A+A_{0}\right) A_{0}^{-1} B^{T} S_{0}^{-1} B A_{0}^{-1}\left(A+A_{0}\right)>0 .
$$

Premultiplying by $\left(A+A_{0}\right) A_{0}^{-1}$ and postmultiplying by $A_{0}^{-1}\left(A+A_{0}\right)$ gives the equivalent condition

$$
(\alpha+\beta) A\left(A+A_{0}\right)^{-1} A_{0}>-\beta B^{T} S_{0}^{-1} B,
$$

or $\left(A_{0}^{-1}+A^{-1}\right)^{-1}>\gamma B^{T} S_{0}^{-1} B$. If this is positive definite then using the same arguments as for the first case above shows that $T<0$. Thus, when $\alpha+\beta>0, \beta<0, \mathcal{P}^{-1} \mathcal{A}$ is not positive definite with respect to $\langle\cdot, \cdot\rangle_{\mathcal{W}}$.

That the combination preconditioned saddle point matrix is indefinite is perhaps unsurprising given the indefiniteness of the $\mathrm{BP}^{+}$and $\mathrm{SZ}^{+}$preconditioned saddle point matrices and we do not consider this combination preconditioner further. What is more startling is that the $\mathrm{BP}^{+}-\mathrm{BD}$ combination preconditioned saddle point matrix described below is positive definite for certain parameters even though separately the $\mathrm{BP}^{+}$and $\mathrm{BD}$ preconditioned saddle point systems are indefinite with respect to inner products. 


\subsection{The $B P^{+}-B D$ combination preconditioner}

Theorem 6 applied to the $\mathrm{BP}^{+}$preconditioner (11) and the BD preconditioner (19), with $c_{1}=-1$, $c_{2}=0, d_{1}=d_{2}=0$ and $\epsilon_{1}=\epsilon_{2}=1$, gives that

$$
\mathcal{P}=\left[\begin{array}{cc}
A_{0} & 0 \\
-\frac{\alpha}{\alpha+\beta} B & \frac{1}{\alpha+\beta} S_{0}
\end{array}\right]
$$

and

$$
\mathcal{W}=\left[\begin{array}{cc}
\alpha\left(A+A_{0}\right)+\beta A_{0} & 0 \\
0 & S_{0}
\end{array}\right]
$$

We note that the cost of one iteration of $\mathcal{W}$-PCG $\left(\mathcal{W}\right.$-PMINRES) with the $\mathrm{BP}^{+}$-BD combination preconditioner is essentially the same as (or is not significantly higher than) the cost of an iteration of $\mathcal{W}$-PCG $\left(\mathcal{W}\right.$-PMINRES) with either the $\mathrm{BP}^{+}$or $\mathrm{BD}$ preconditioner on its own.

As we shall see, for this choice of $\mathcal{P}$ and $\mathcal{W}$ there $d o$ exist $\alpha$ and $\beta$ for which $\mathcal{P}^{-1} \mathcal{A}$ is positive definite with respect to an inner product.

\section{Theorem 8}

Let (1) be left preconditioned by the $\mathrm{BP}^{+}$-BD combination preconditioner (44), so that $\mathcal{P}^{-1} \mathcal{A}$ is self-adjoint with respect to $\langle\cdot, \cdot\rangle_{\mathcal{W}}$, where $\mathcal{W}$ is defined by (45).

When

I. $\alpha>0$ and $\alpha+\beta<0, \mathcal{W}$ defines an inner product, with respect to which $\mathcal{P}^{-1} \mathcal{A}$ is positive definite, if and only if

$$
A_{0}<-\frac{\alpha}{\alpha+\beta} A
$$

II. $\alpha>0$ and $\alpha+\beta>0, \mathcal{W}$ defines an inner product with respect to which $\mathcal{P}^{-1} \mathcal{A}$ is indefinite;

III. $\alpha<0$ and $\alpha+\beta>0, \mathcal{W}$ defines an inner product if and only if

$$
A_{0}>-\frac{\alpha}{\alpha+\beta} A
$$

but $\mathcal{P}^{-1} \mathcal{A}$ is indefinite with respect to this inner product;

IV. $\alpha<0$ and $\alpha+\beta<0, \mathcal{W}$ does not define an inner product.

Proof

Recall from (4) that $\mathcal{P}^{-1} \mathcal{A}$ is $\mathcal{W}$-positive definite if and only if $\mathcal{W} \mathcal{P}^{-1} \mathcal{A}>0$, where

$$
\begin{aligned}
\mathcal{W P}^{-1} \mathcal{A} & =\left[\begin{array}{cc}
\alpha A A_{0}^{-1}\left(A+A_{0}\right)+\beta A & \alpha A A_{0}^{-1} B^{T}+(\alpha+\beta) B^{T} \\
\alpha B A_{0}^{-1}\left(A+A_{0}\right)+\beta B & \alpha B A_{0}^{-1} B^{T}-(\alpha+\beta) C
\end{array}\right] \\
& =\left[\begin{array}{cc}
I & 0 \\
B A^{-1} & I
\end{array}\right]\left[\begin{array}{cc}
M & 0 \\
0 & T-B A^{-1} M A^{-1} B^{T}
\end{array}\right]\left[\begin{array}{cc}
I & A^{-1} B^{T} \\
0 & I
\end{array}\right],
\end{aligned}
$$

with

$$
M=\alpha A A_{0}^{-1} A+(\alpha+\beta) A=A\left(\alpha A_{0}^{-1}+(\alpha+\beta) A^{-1}\right) A
$$

and $T=\alpha B A_{0}^{-1} B^{T}-(\alpha+\beta) C$.

From (46) and Sylvester's law of inertia it follows that $\mathcal{P}^{-1} \mathcal{A}$ is $\mathcal{W}$-positive definite when $M>0$ and $T>B A^{-1} M A^{-1} B^{T}$. The latter condition is equivalent to requiring that

$$
\alpha B A_{0}^{-1} B^{T}-(\alpha+\beta) C>\alpha B A_{0}^{-1} B^{T}+(\alpha+\beta) B A^{-1} B^{T}
$$

or that

$$
-(\alpha+\beta) C>(\alpha+\beta) B A^{-1} B^{T}
$$




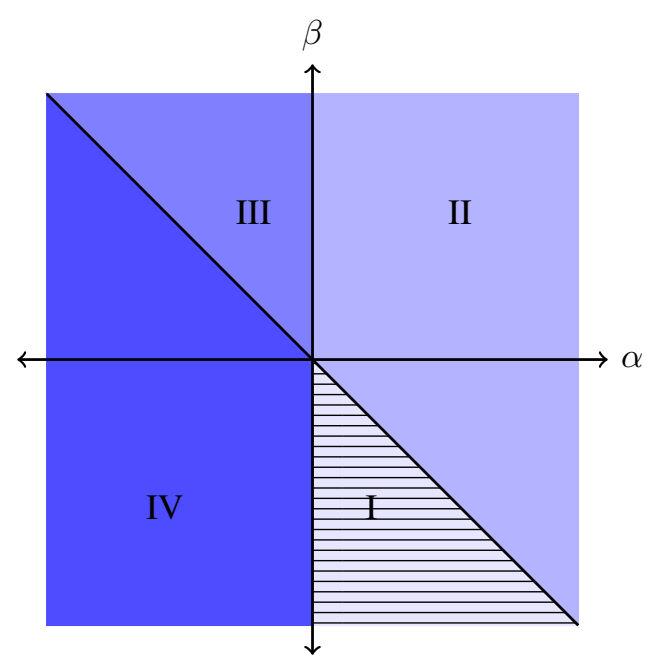

Figure 2. Regions (distinguished by different colours) that are considered in Theorem 8 for the $\mathrm{BP}^{+}-\mathrm{BD}$ combination preconditioner. The horizontal lines show where $\mathcal{P}^{-1} \mathcal{A}$ can be made positive definite with respect to $\langle\cdot, \cdot\rangle_{\mathcal{W}}$ by choosing $\alpha$ and $\beta$ appropriately or by scaling $A_{0}$ and $S_{0}$.

The conditions for positive definiteness of $\mathcal{P}^{-1} \mathcal{A}$ with respect to $\langle\cdot, \cdot\rangle_{\mathcal{W}}$ are, therefore, that $M>0$ and (48) is satisfied, which we consider for each case in Figure 2.

I. If $\alpha+\beta<0$ and $\alpha>0, \mathcal{W}$ is positive definite if and only if

$$
A>-\frac{\alpha+\beta}{\alpha} A_{0}
$$

This is also the condition for positive definiteness of $M$ in (47). Now, (48) is equivalent to $C>-B A^{-1} B^{T}$, the left-hand side of which is symmetric positive semidefinite and the right-hand side of which is negative definite. Thus, this inequality is always satisfied and we have that when $\alpha+\beta\left\langle 0, \alpha>0\right.$ and $\mathcal{W}$ defines an inner product, $\mathcal{P}^{-1} \mathcal{A}$ is $\mathcal{W}$-positive definite.

II. When $\alpha>0$ and $\alpha+\beta>0, \mathcal{W}$ and $M$ are positive definite. However, since $C$ is positive semidefinite, $A$ is positive definite and $B$ has full rank, (48) cannot hold. Indeed, $T-$ $B A^{-1} M A^{-1} B^{T}<0$ and $\mathcal{P}^{-1} \mathcal{A}$ is indefinite with respect to $\langle\cdot, \cdot\rangle_{\mathcal{W}}$.

III. When $\alpha<0$ with $\alpha+\beta>0, \mathcal{W}$ defines an inner product if and only if

$$
\frac{1}{\alpha+\beta} A<-\frac{1}{\alpha} A_{0}
$$

Furthermore, $M$ in (47) is positive definite if and only if $\alpha A_{0}^{-1}+(\alpha+\beta) A^{-1}>0$ which, since $A_{0}$ and $A$ are positive definite, is equivalent to

$$
\frac{1}{\alpha+\beta} A<-\frac{1}{\alpha} A_{0}
$$

It follows that $M>0$ whenever $\mathcal{W}>0$. However, as in Case II, (48) is not satisfied and $T-$ $B A^{-1} M A^{-1} B^{T}<0$. Thus, when $\alpha+\beta>0$ and $\mathcal{W}$ defines an inner product it is one with respect to which $\mathcal{P}^{-1} \mathcal{A}$ is indefinite.

IV. If $\alpha<0$ and $\alpha+\beta<0, \mathcal{W}$ is indefinite and does not define an inner product.

The pivotal result of Theorem 8 is that when $\alpha+\beta<0$ with $\alpha>0$ it is possible to obtain from the $\mathrm{BP}^{+}$and $\mathrm{BD}$ preconditioners-for which the preconditioned saddle point matrices are indefinite with respect to inner products-a combination preconditioner for which $\mathcal{P}^{-1} \mathcal{A}$ is selfadjoint and positive definite with respect to a nonstandard inner product. One of the advantages 
of positive definiteness is that $\mathcal{W}$-PCG may be reliably applied instead of $\mathcal{W}$-PMINRES. Even for $\mathcal{W}$-PMINRES, the eigenvalues of a $\mathcal{W}$-positive definite preconditioned saddle point matrix lie on the positive real line and, if clustered, might lead to faster convergence than can be achieved for an indefinite system. Indeed, we shall see below that for these parameters convergence of the combination preconditioner for both $\mathcal{W}$-PMINRES and $\mathcal{W}$-PCG is rapid.

Regardless of whether $\mathcal{W}$-PMINRES or $\mathcal{W}$-PCG is applicable, convergence for the $\mathrm{BP}^{+}$-BD preconditioned system depends heavily on the eigenvalues of $\mathcal{P}^{-1} \mathcal{A}$. These can be bounded when $C=0$, as the following theorem shows.

\section{Theorem 9}

Let (1), with $C=0$ be left preconditioned by the $\mathrm{BP}^{+}$-BD combination preconditioner (44) and let $\mathcal{W}$ in (45) be positive definite, so that $\mathcal{P}^{-1} \mathcal{A}$ is self-adjoint with respect to an inner product. Additionally, let $\delta$ and $\Delta$ be such that

$$
0<\delta \leq \psi=\frac{y^{T} A_{0} y}{y^{T} A y} \leq \Delta
$$

for all $y \in \mathbb{R}^{n}$ and let $\omega$ be such that

$$
0 \leq \omega=\frac{z^{T} B^{T} S_{0}^{-1} B z}{z^{T} A z} \leq \phi
$$

for all $z \in \mathbb{R}^{n}$.

Let $\lambda$ be an eigenvalue of $\mathcal{P}^{-1} \mathcal{A}$ with corresponding eigenvector $u=\left[u_{1}^{T}, u_{2}^{T}\right], u_{1} \in \mathbb{R}^{n}, u_{2} \in$ $\mathbb{R}^{m}$. Then if $B u_{1}=0$,

$$
\frac{1}{\Delta} \leq \lambda^{+} \leq \frac{1}{\delta}
$$

Otherwise, when

I. $\alpha>0$ but $\alpha+\beta<0$ the remaining eigenvalues $\lambda^{+}$, which are all positive, satisfy

$$
\frac{1}{\Delta} \leq \lambda^{+} \leq \frac{(1+\alpha \phi)+\sqrt{(1+\alpha \phi)^{2}+4 \delta \phi(\alpha+\beta)}}{2 \delta}
$$

or

$$
\zeta_{1}<\lambda^{+} \leq \frac{(1+\alpha \phi)-\sqrt{(1+\alpha \phi)^{2}+4 \delta \phi(\alpha+\beta)}}{2 \delta}
$$

II and III. $\alpha+\beta>0$, the remaining positive eigenvalues $\lambda^{+}$of $\mathcal{P}^{-1} \mathcal{A}$ satisfy

$$
\frac{1}{\Delta} \leq \lambda^{+} \leq \frac{(1+\alpha \phi)+\sqrt{(1+\alpha \phi)^{2}+4 \delta \phi(\alpha+\beta)}}{2 \delta}
$$

while negative eigenvalues $\lambda^{-}$are bounded by

$$
\frac{(1+\alpha \phi)-\sqrt{(1+\alpha \phi)^{2}+4 \delta \phi(\alpha+\beta)}}{2 \delta} \leq \lambda^{-}<\zeta_{2},
$$

where $\zeta_{1}, \zeta_{2}>0$ are real constants.

Proof

The $\mathrm{BP}^{+}$-BD combination preconditioner is a Krzyżanowski preconditioner with $c=-\frac{\alpha}{\alpha+\beta}, d=0$ and $S_{0}$ replaced by $\frac{1}{\alpha+\beta} S_{0}$. Thus, by Lemma $4, \lambda \neq 0,-\frac{\alpha+\beta}{\alpha}$ and $u \neq 0$. Additionally, when $B u_{1}=0$,

$$
\lambda=\frac{u_{1}^{T} A u_{1}}{u_{1}^{T} A_{0} u_{1}}
$$


and (49) then implies that

$$
\frac{1}{\Delta} \leq \lambda \leq \frac{1}{\delta}
$$

If $B u_{1} \neq 0$ then , by Lemma 4 ,

$$
\lambda=\frac{(1+\alpha \omega) \pm \sqrt{(1+\alpha \omega)^{2}+4 \psi \omega(\alpha+\beta)}}{2 \psi},
$$

where we have multiplied the top and bottom of (24) by $\alpha+\beta$. These roots must be real since $\mathcal{P}^{-1} \mathcal{A}$ is self-adjoint with respect to an inner product. However, it is possible, albeit tedious, to verify this using the condition that $\alpha\left(A+A_{0}\right)+\beta A_{0}>0$. Cases I-III in Figure 2 require separate examination to determine bounds on the eigenvalues. We present the proof for one case here; proofs for the remaining two are similar.

I. Let $\alpha>0$ and $\alpha+\beta<0$. Both roots in (55) are positive, since $\alpha+\beta$ is negative, $\phi$ is positive and $\omega$ is nonnegative. The larger root is given by

$$
\lambda=\frac{(1+\alpha \omega)+\sqrt{(1+\alpha \omega)^{2}+4 \psi \omega(\alpha+\beta)}}{2 \psi}
$$

which we see, by considering (49) and (50), is minimized when $\omega=0$ and $\psi=\Delta$ and so $\lambda \geq \frac{1}{\Delta}$. Conversely, (56) is maximized when $\omega=\phi$ and $\psi=\delta$ which gives that

$$
\frac{1}{\Delta} \leq \lambda \leq \frac{(1+\alpha \phi)+\sqrt{(1+\alpha \phi)^{2}+4 \delta \phi(\alpha+\beta)}}{2 \delta} .
$$

The smaller root of (55) is

$$
\lambda=\frac{(1+\alpha \omega)-\sqrt{(1+\alpha \omega)^{2}+4 \psi \omega(\alpha+\beta)}}{2 \psi},
$$

which is also minimized when $\omega=0$ and $\psi=\Delta$. This implies that $\lambda \geq 0$. However, we concluded previously that $\lambda \neq 0$ and we must have that $\lambda>0$. Additionally, (57) is is maximized by choosing $\psi=\delta$ and $\omega=\phi$ which gives that

$$
\lambda \leq \frac{(1+\alpha \phi)-\sqrt{(1+\alpha \phi)^{2}+4 \delta \phi(\alpha+\beta)}}{2 \delta} .
$$

We now wish to improve on the bound $\lambda>0$ on the smaller root of (55). To do so, we follow [?] and consider the generalized eigenvalue problem $\mathcal{P}_{D}^{-\frac{1}{2}} \mathcal{A} \mathcal{P}_{D}^{-\frac{1}{2}} v=\lambda \mathcal{P}_{D}^{-\frac{1}{2}} \mathcal{P} \mathcal{P}_{D}^{-\frac{1}{2}} v$, where

$$
\mathcal{P}_{D}=\left[\begin{array}{cc}
A_{0} & 0 \\
0 & S_{0}
\end{array}\right]
$$

and $v=\mathcal{P}_{D}^{\frac{1}{2}} u=\left[v_{1}^{T}, v_{2}^{T}\right]^{T}$. This can be expressed as $\widetilde{\mathcal{A}} v=\lambda \widetilde{\mathcal{P}} v$, where

$$
\widetilde{\mathcal{A}}=\left[\begin{array}{cc}
\widetilde{A} & \widetilde{B}^{T} \\
\widetilde{B} & 0
\end{array}\right], \quad \widetilde{\mathcal{P}}=\left[\begin{array}{cc}
I & 0 \\
-\frac{\alpha}{\alpha+\beta} \widetilde{B} & \frac{1}{\alpha+\beta} I
\end{array}\right],
$$

$\widetilde{A}=A_{0}^{-\frac{1}{2}} A A_{0}^{-\frac{1}{2}}$ and $\widetilde{B}=S_{0}^{-\frac{1}{2}} B A_{0}^{-\frac{1}{2}}$. In component form, we have that

$$
\begin{aligned}
\widetilde{A} v_{1}+\widetilde{B}^{T} v_{2} & =\lambda v_{1} \\
(\alpha+\beta+\lambda \alpha) \widetilde{B} v_{1} & =\lambda v_{2} .
\end{aligned}
$$


where $v_{2} \neq 0$ (since then $v_{1}=0$ as in Lemma 4). Additionally, if $v_{2}=0,(\alpha+\beta+\lambda \alpha) \tilde{B} v_{1}=0$ which, since $\lambda \neq(\alpha+\beta) / \alpha$ implies that $S_{0}^{-\frac{1}{2}} B u_{1}=0$ or that $B u_{1}=0$. However, we are interested only in the case that $B u_{1} \neq 0$ and so we can assume that $v_{1} \neq 0 \neq v_{2}$.

Premultiplying (58) by $v_{1}^{*}$ and rearranging shows that $v_{1}^{*} \widetilde{B}^{T} v_{2}=\lambda\left\|v_{1}\right\|_{2}^{2}-v_{1}^{*} \widetilde{A} v_{1}$. Taking the conjugate transpose of (59), postmultiplying by $v_{2}$ and substituting for $v_{1}^{*} \widetilde{B}^{T} v_{2}$ gives the quadratic equation

$$
\alpha\left\|v_{1}\right\|_{2}^{2} \lambda^{2}+\left[(\alpha+\beta)\left\|v_{1}\right\|_{2}^{2}-\alpha v_{1}^{*} \widetilde{A} v_{1}-\left\|v_{2}\right\|_{2}^{2}\right] \lambda-(\alpha+\beta) v_{1}^{*} \widetilde{A} v_{1}=0 .
$$

Now, for any quadratic equation $a x^{2}+b x+c=0$ with roots $\lambda_{1}, \lambda_{2}$ such that $\lambda_{1}>\lambda_{2}, \lambda_{2}=$ $c /\left(a \lambda_{1}\right)$, which shows that $\lambda_{2}$ is bounded away from the origin provided $c /\left(a \lambda_{1}\right)$ is bounded by positive constants above and below. We know from (52) that the larger root $\lambda_{2}$ is bounded. Additionally,

$$
\frac{c}{a}=\left(-\frac{\alpha+\beta}{\alpha}\right) \frac{v_{1}^{*} \widetilde{A} v_{1}}{\left\|v_{1}\right\|_{2}^{2}}
$$

and from (49) it is clear that

$$
0<\left(-\frac{\alpha+\beta}{\alpha}\right) \frac{1}{\Delta} \leq(\alpha+\beta) \frac{v_{1}^{*} \widetilde{A} v_{1}}{\left\|v_{1}\right\|_{2}^{2}} \leq\left(-\frac{\alpha+\beta}{\alpha}\right) \frac{1}{\delta} .
$$

Thus, the smaller root $\lambda_{2}$ is bounded away from the origin, i.e.,

$$
0<\zeta_{1} \leq \lambda \leq \frac{(1+\alpha \phi)-\sqrt{(1+\alpha \phi)^{2}+4 \delta \phi(\alpha+\beta)}}{2 \delta}
$$

for some $\zeta_{1}$. Bounds for Cases II and III are similarly obtained.

Remark 2. Neither (53) nor (54) bound the eigenvalues of $\mathcal{P}^{-1} \mathcal{A}$ away from the origin. The difficulty is caused by (50). However, it may be possible to bound the eigenvalues away from the origin for certain applications.

If it is possible to obtain bounds of the form (49) and (50) without too great an expense we can ascertain a priori the eigenvalue distribution of $\mathcal{P}^{-1} \mathcal{A}$. This often determines the convergence of both $\mathcal{W}$-PMINRES and $\mathcal{W}$-PCG. Moreover, it may be possible to use these bounds to choose $\alpha$ and $\beta$ (and scale $A_{0}$ and $S_{0}$ if necessary) to obtain good eigenvalues.

\section{NUMERICAL EXAMPLES}

The four test problems to which we apply the $\mathrm{BP}^{+}$- $\mathrm{BD}$ combination preconditioner are the Stokes problems in [?, Chapter 5], discretized by Taylor-Hood $\left(Q_{2}-Q_{1}\right)$ finite elements by the Matlab package IFISS [?]. Since Taylor-Hood elements are stable, $C=0$ in (1). The approximation $A_{0}$ is one AMG V-cycle computed by the HSL MI20 routine [?] with the MATLAB interface while the Schur complement is approximated by the pressure mass matrix computed by IFISS. Since $\mathcal{P}_{\text {comb }}^{-1} \mathcal{A}$ can be made positive definite with respect to an inner product, we apply both $\mathcal{W}$-PMINRES and $\mathcal{W}$-PCG to the combination preconditioned system, while $\mathcal{W}$-PMINRES is used to solve separately the $\mathrm{BP}^{+}$and $\mathrm{BD}$ preconditioned systems. Since, as noted in Section 5.2, the cost per iteration of $\mathcal{W}$-PCG (or $\mathcal{W}$-PMINRES) with each of the $\mathrm{BP}, \mathrm{BD}$ and $\mathrm{BP}^{+}$-BD preconditioners is essentially the same it is fair to compare the number of iterations required for convergence.

The termination criterion for both methods it that the relative preconditioned residual, measured in the Euclidean norm, is reduced by a factor of $10^{-6}$. We perform $\mathcal{W}$-PCG and $\mathcal{W}$-PMINRES for values of $\alpha$ and $\beta$ between -2 and 2 in steps of 0.1 , neglecting those that cause $\mathcal{P}$ to be singular. The 


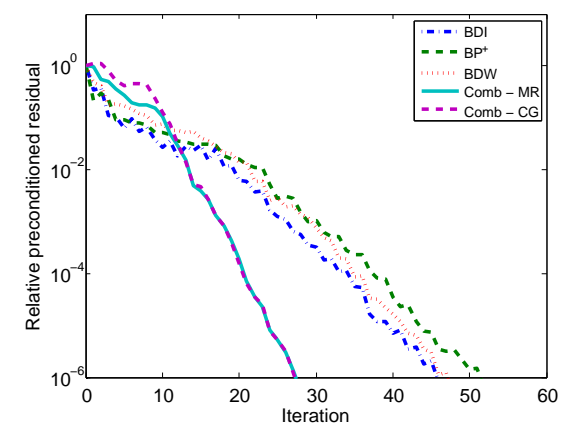

(a) backward step

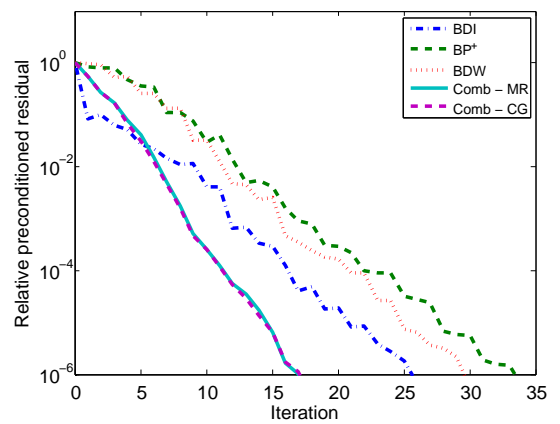

(b) regularized cavity

Figure 3. Convergence plots for $\mathrm{BP}^{+}$-BD combination preconditioned $\mathcal{W}$-PMINRES and $\mathcal{W}$-PCG for (a) the backward step flow with $\alpha=1.1$ and $\beta=-2$ and (b) the regularized cavity flow with $\alpha=1.1$ and $\beta=-2$. The plots also show convergence of $\mathrm{BP}^{+}$preconditioned $\mathcal{W}$-PMINRES, BD preconditioned $\mathcal{W}$-PMINRES and block diagonal preconditioned $I$-PMINRES.

$(\alpha, \beta) \in[-2,2] \times[-2,2]$ pairs shown in tables in this chapter are those that give the lowest number of iterations, possibly subject to $\mathcal{W}_{\text {comb }}$ defining and inner product and, for $\mathcal{W}$-PCG, when $\mathcal{P}_{\text {comb }} \mathcal{A}$ being positive definite with respect to $\langle\cdot, \cdot\rangle_{\mathcal{W}_{\text {comb }}}$. To determine whether $\mathcal{W}_{\text {comb }}$ is positive definite we compute $A_{0}$ explicitly. Clearly, this is infeasible in practice for large problems. Our numerical experiments indicate that the optimal choice of $\alpha$ and $\beta$ is fairly mesh independent, so that good choices could be obtained by examining a smaller problem. More sophisticated strategies, perhaps based on Fourier analysis of an ideal problem, could also be employed but we have not investigated this.

We have tabulated in Tables II and III the lowest iteration count for the combination preconditioner for which $\mathcal{W}_{\text {comb }}$ defines an inner product and, for the conjugate gradient method, for which $\mathcal{P}^{-1} \mathcal{A}$ is positive definite with respect to this inner product. For most problems this count is obtained by multiple choices of $\alpha$ and $\beta$. It is clear that the combination preconditioner offers superior performance to either the $\mathrm{BP}^{+}$preconditioner or the $\mathrm{BD}$ preconditioner. The relative reduction in the number of iterations required by the combination preconditioner, in comparison to the better performing of the $\mathrm{BD}$ and $\mathrm{BP}^{+}$preconditioners, is $40.1 \%$ on average for $\mathcal{W}$-PMINRES and $40.3 \%$ on average for $\mathcal{W}$-PCG. The lowest iteration count for all problems with $\mathcal{W}$-PMINRES is achieved with $(\alpha, \beta)=(1.1,-2)$, indicating that this choice is optimal for these problems. For $\mathcal{W}$-PCG, the best choice of $\alpha$ and $\beta$ is also fairly mesh independent. Additionally, in many cases the optimal choices of $\alpha$ and $\beta$ for $\mathcal{W}$-PMINRES and $\mathcal{W}$-PCG coincide and the number of iterations for each method is close. This suggests that $\mathcal{W}$-PMINRES performs best when $\mathcal{P}^{-1} \mathcal{A}$ is $\mathcal{W}_{\text {comb }}{ }^{-}$ positive definite. It also appears that for these problems the cheaper $\mathcal{W}$-PCG method is preferable to $\mathcal{W}$-PMINRES.

Convergence plots for the backward step and regularized cavity flows with $h=2^{-4}$ for the values of $\alpha$ and $\beta$ listed in Table II are shown in Figure 3. We observe that the $\mathcal{W}$-PCG and $\mathcal{W}$-PMINRES curves are very similar. Both decrease more rapidly than those of $\mathrm{BP}^{+}$and $\mathrm{BD}$ preconditioned $\mathcal{W}$-MINRES and of block-diagonal preconditioned $I$-PMINRES (with $\mathcal{P}$ given by (19)) .

Although optimal PMINRES convergence occurs when $\mathcal{W}_{c o m b}$ defines an inner product, faster convergence can sometimes be observed for the conjugate gradient method when $\mathcal{W}_{\text {comb }}$ is not positive definite, as can be seen from Table III and Figure 3. The average relative reduction when compared with the better of $\mathrm{BW}-$ or $\mathrm{BP}^{+}-\mathrm{PMINRES}$ is slightly higher, at $44.1 \%$. However, robustness is not guaranteed when $\langle\cdot, \cdot\rangle_{\mathcal{W}_{\text {comb }}}$ is not an inner product and we caution that the $\mathrm{BP}^{+}$BD combination-PCG method may fail for other approximations of $A_{0}$ or $S_{0}$ or for different saddle point problems. 


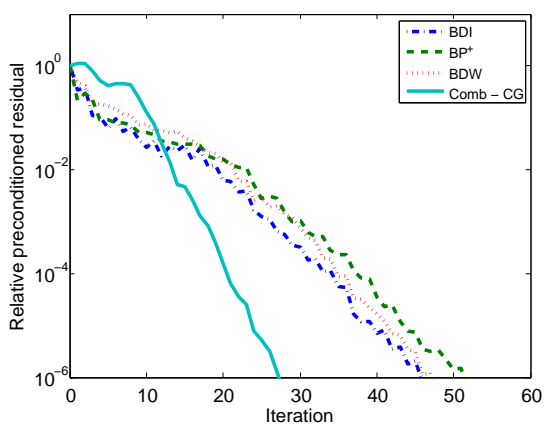

(a) backward step

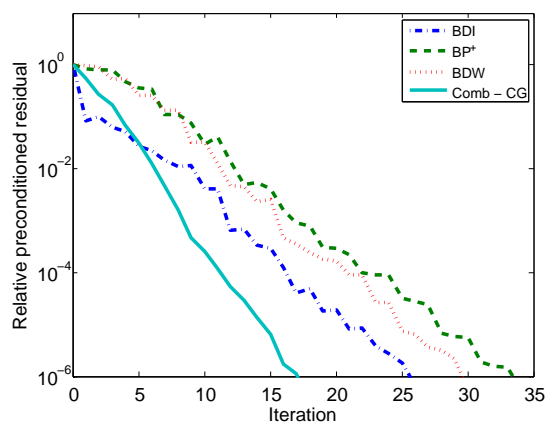

(b) regularized cavity

Figure 4. Convergence plots for $\mathrm{BP}^{+}$-BD combination preconditioned $\mathcal{W}$-PCG for (a) the backward step flow with $\alpha=1$ and $\beta=-2$ and (b) the regularized cavity flow with $\alpha=1$ and $\beta=-1.9$. The plots also show convergence of $\mathrm{BP}^{+}$preconditioned $\mathcal{W}$-PMINRES, BD preconditioned $\mathcal{W}$-PMINRES and block diagonal preconditioned $I$-PMINRES. Note that $\mathcal{W}$ is not positive definite for these parameter choices.

We additionally examined the effect of scaling the $\mathrm{BP}^{+}$and $\mathrm{BD}$ preconditioners separately, by setting $\beta$ or $\alpha$ to zero and varying the other parameter. Both preconditioners were sensitive to scaling, although the optimal scaling appeared to be problem dependent.

\section{CONCLUSIONS}

The Krzyżanowski preconditioner provides a useful framework for examining preconditioners that render a symmetric saddle point matrix self-adjoint with respect to an inner product. We have derived expressions for the eigenvalues of the Krzyżanowski preconditioned saddle point matrix, when $C=0$, in terms of certain generalized Rayleigh quotients. These can be used to obtain bounds on the eigenvalues for specific instances of the Krzyżanowski preconditioner that can, in turn, either help to predict the convergence of $\mathcal{W}$-PCG or $\mathcal{W}$-PMINRES applied to the saddle point system or to tune the preconditioner for optimal performance. We have also proved that the $\mathrm{SZ}^{+}$preconditioned saddle point matrix that is always self-adjoint with respect to an inner product $\langle\cdot, \cdot\rangle_{\mathcal{W}}$ but is never $\mathcal{W}$-positive definite.

Expressions for combinations of certain Krzyżanowski preconditioners have been derived. From these we constructed two combination preconditioners, for each of which the constituent preconditioners were such that the preconditioned saddle point matrix was not positive definite with respect to an inner product. The $\mathrm{BP}^{+}-\mathrm{SZ}^{+}$combination preconditioned saddle point matrix is never positive definite with respect to an inner product. However, surprisingly, the $\mathrm{BP}^{+}$-BD combination preconditioned saddle point matrix is positive definite with respect to an inner product for certain parameter choices. This means that a $\mathcal{W}$-PCG method may be applied to the preconditioned system, iterations of which are cheaper than those of $\mathcal{W}$-PMINRES. More importantly, it highlights the power of combination preconditioning, which constructs a preconditioner $\mathcal{P}$ from two preconditioners, $\mathcal{P}_{1}$ and $\mathcal{P}_{2}$, such that $\mathcal{P}^{-1} \mathcal{A}$ can be made positive definite with respect to an inner product when neither $\mathcal{P}_{1}^{-1} \mathcal{A}$ nor $\mathcal{P}^{-1} \mathcal{A}$ are.

The $\mathrm{BP}^{+}-\mathrm{BD}$ combination preconditioner can, additionally, be more efficient than either the $\mathrm{BP}^{+}$or $\mathrm{BD}$ preconditioners and performs well when the combination preconditioned saddle point matrix is self-adjoint and positive definite with respect to an inner product. Even faster $\mathcal{W}$-PCG convergence is observed when $\mathcal{W}$ does not define an inner product for the particular problems examined here, although this may not always occur and the reliability of the $\mathcal{W}$-PCG method cannot be guaranteed in this case. 
The $\mathrm{BP}^{+}-\mathrm{BD}$ combination preconditioner shows that combination preconditioning can be used to achieve positive definiteness. It would certainly be interesting to determine other combinations for which this also occurs.

\section{ACKNOWLEDGEMENT}

We are indebted to the anonymous referee for helpful suggestions and remarks. 
Table I. Krzyżanowski parameters for saddle point preconditioners. Here $\widehat{A}$ and $\widehat{S}$ represent (typically problem-dependent) approximations of $A$ and the Schur complement $S$.

\begin{tabular}{lrrrrr}
\hline Preconditioner & $A_{0}$ approximation & $S_{0}$ approximation & $c$ & $d$ & $\epsilon$ \\
\hline Block diagonal & $\widehat{A}$ & $\widehat{S}$ & 0 & 0 & 1 \\
Bramble-Pasciak & $\widehat{A}$ & $-I$ & 1 & 0 & -1 \\
$\mathrm{BP}^{+}$ & $\widehat{A}$ & $\widehat{S}$ & -1 & 0 & 1 \\
$\mathrm{SZ}^{+}$ & $\widehat{A}$ & $-\widehat{S}$ & 1 & 1 & 1 \\
$\mathrm{SZ}^{+}$ & $\widehat{A}$ & $\widehat{S}$ & -1 & -1 & 1 \\
\hline
\end{tabular}

Table II. Iteration counts for the $\mathrm{BP}^{+}$preconditioner, the $\mathrm{BD}$ preconditioner and best possible $\mathrm{BP}^{+}-\mathrm{BD}$ combination preconditioner for $\mathcal{W}$-PMINRES. Also included is the percentage reduction in the number of iterations required by the combination preconditioner compared with the better performing of the $\mathrm{BP}^{+}$and BD preconditioners.

\begin{tabular}{llcccc}
\hline Problem & $h$ & $\mathrm{BP}^{+}$ & $\mathrm{BD}$ & $\operatorname{Comb}(\alpha, \beta)$ & \% reduction \\
\hline \multirow{3}{*}{ Channel flow } & $2^{-3}$ & 31 & 29 & $18(1.1,-2)$ & 38 \\
& $2^{-4}$ & 36 & 33 & $19(1.1,-2)$ & 42 \\
& $2^{-5}$ & 39 & 34 & $20(1.1,-2)$ & 41 \\
Backward step & $2^{-3}$ & 47 & 43 & $25(1.1,-2)$ & 42 \\
& $2^{-4}$ & 52 & 48 & $28(1.1,-2)$ & 42 \\
& $2^{-5}$ & 53 & 50 & $28(1.1,-2)$ & 44 \\
Cavity flow & $2^{-3}$ & 30 & 26 & $15(1.1,-2)$ & 42 \\
& $2^{-4}$ & 34 & 30 & $18(1.1,-2)$ & 40 \\
Colliding flow & $2^{-5}$ & 35 & 32 & $18(1.1,-2)$ & 44 \\
& $2^{-3}$ & 23 & 24 & $15(1.1,-2)$ & 35 \\
& $2^{-4}$ & 29 & 26 & $17(1.1,-2)$ & 35 \\
\hline
\end{tabular}

Table III. Iteration counts for the $\mathrm{BP}^{+}$preconditioner, the $\mathrm{BD}$ preconditioner and best possible $\mathrm{BP}^{+}$$\mathrm{BD}$ combination preconditioner for $\mathcal{W}$-PCG. Also included is the percentage reduction in the number of iterations required by the combination preconditioner compared with the better performing of the $\mathrm{BP}^{+}$and BD preconditioners.

\begin{tabular}{|c|c|c|c|c|c|c|c|}
\hline \multirow[b]{2}{*}{ Problem } & \multirow[b]{2}{*}{$h$} & \multirow[b]{2}{*}{$\mathrm{BP}^{+}$} & \multirow[b]{2}{*}{$\mathrm{BD}$} & \multicolumn{2}{|c|}{$\mathcal{W}$-PCG: $\mathcal{W}>0$} & \multicolumn{2}{|c|}{$\mathcal{W}$-PCG: $\mathcal{W} \ngtr 0$} \\
\hline & & & & $\operatorname{Comb}(\alpha, \beta)$ & $\%$ reduction & Comb $(\alpha, \beta)$ & $\%$ reduction \\
\hline \multirow{3}{*}{ Channel flow } & $2^{-3}$ & 31 & 29 & $17(1.1,-2)$ & 41 & $17(1,-2)$ & 41 \\
\hline & $2^{-4}$ & 36 & 33 & $19(1.1,-2)$ & 42 & $17(0.9,-1.8)$ & 48 \\
\hline & $2^{-5}$ & 39 & 34 & $20(1.1,-2)$ & 41 & $20(1,-2)$ & 41 \\
\hline \multirow{3}{*}{ Backward step } & $2^{-3}$ & 47 & 43 & $25(1.1,-2)$ & 42 & $24(0.9,-1.9)$ & 44 \\
\hline & $2^{-4}$ & 52 & 48 & $28(1.1,-2)$ & 42 & $25(1,-2)$ & 48 \\
\hline & $2^{-5}$ & 53 & 50 & $18(1,-1.8)$ & 44 & $17(1,-2)$ & 47 \\
\hline \multirow{3}{*}{ Cavity flow } & $2^{-3}$ & 30 & 26 & $15(0.8,-1.5)$ & 42 & $14(1,-2)$ & 46 \\
\hline & $2^{-4}$ & 34 & 30 & $18(1.1,-2)$ & 40 & $16(1,-1.9)$ & 47 \\
\hline & $2^{-5}$ & 35 & 32 & $20(0.8,-1.4)$ & 38 & $17(1,-2)$ & 47 \\
\hline \multirow{3}{*}{ Colliding flow } & $2^{-3}$ & 23 & 24 & $15(1.1,-2)$ & 35 & $14(1,-2)$ & 39 \\
\hline & $2^{-4}$ & 29 & 26 & $16(1.1,-2)$ & 38 & $15(1,-2)$ & 42 \\
\hline & $2^{-5}$ & 29 & 28 & $17(1.1,-2)$ & 39 & $17(1.1,-2)$ & 39 \\
\hline
\end{tabular}

